\title{
ON MAPPINGS WHOSE INVERSES SATISFY THE POLETSKY INEQUALITY
}

\author{
Evgeny Sevost'yanov and Sergei Skvortsov
}

\author{
Zhytomyr Ivan Franko State University \\ 40 Bol'shaya Berdichevskaya Str., 10008 Zhytomyr, Ukraine \\ and Institute of Applied Mathematics and Mechanics of NAS of Ukraine \\ 1 Dobrovol'skogo Str., 84100 Slov'yans'k, Ukraine; esevostyanov2009@gmail.com \\ Zhytomyr Ivan Franko State University \\ 40 Bol'shaya Berdichevskaya Str., 10008 Zhytomyr, Ukraine; serezha.skv@gmail.com
}

\begin{abstract}
The article investigates mappings whose inverses distort the modulus of paths similarly to the Poletsky inequality. It is proved that the classes of such mappings form equicontinuous families if the majorant corresponding to the distortion of the module is integrable in the domain of their definition. Under additional conditions on the geometry of the domain of definition and the image domain these families are equicontinuous, not only at inner, but also at boundary points. In addition, the question of removability of the isolated singularities for such mappings is resolved.
\end{abstract}

\section{Introduction}

In the theory of quasiconformal mappings, there are several remarkable results relating to the equicontinuity of families of mappings. In particular, the classical Väisälä result asserts that families of such mappings are equicontinuous if they do not take at least two fixed values in an extended Euclidean space, see [Va, Theorem 19.2]. Many other results on this topic are also known. In particular, there are analogues of the Väisälä theorem relating to mappings with branching, mappings between fixed domains and mappings with unbounded characteristic, see e.g. [MRV, Theorem 3.17], [ $\mathrm{NP}_{1}$, Theorem 3.1], [ $\mathrm{NP}_{2}$, Theorem 3.1], [Cr, Theorem 8.9], [GU, Theorem A] and [MRSY 2 , Theorem 3.1, Corollary 3.6]. In particular, the following result holds; see [MRV, Theorem 3.17].

Theorem. (Martio-Rickman-Väisälä) Suppose that $G$ is a domain in $\overline{\mathbf{R}^{n}}$ and $F \subset \overline{\mathbf{R}^{n}}$ is a compact set of a positive capacity. Then for every $K \geqslant 1$, the family of all $K$-quasimeromorphic mappings $f: G \rightarrow \overline{\mathbf{R}^{n}} \backslash F$ is equicontinuous with respect to the chordal metric.

We also give another classical result on the global behavior of maps, see $\left[\mathrm{NP}_{1}\right.$, Theorem 3.1].

Theorem. (Näkki-Palka) Let $\mathfrak{F}$ be a family of $K$-quasiconformal mappings of a domain $D \neq \overline{\mathbf{R}^{n}}$ onto a domain $D^{\prime}$ and let either $D$ or $D^{\prime}$ be quasiconformally collared on the boundary. Then $\mathfrak{F}$ is uniformly equicontinuous if and only if each $f \in \mathfrak{F}$ can be extended to a continuous mapping of $D$ onto $D^{\prime}$ and $\inf _{\mathfrak{F}} h(f(A))>0$ for some continuum $A$ in $D$.

https://doi.org/10.5186/aasfm.2020.4520

2010 Mathematics Subject Classification: Primary 30C65; Secondary 32U20, 31B15.

Key words: Mappings with finite and bounded distortion, quasiconformal mappings, local and boundary behavior. 
Here $h(E)$ denotes the chordal diameter of the set $E$ in the extended Euclidean space. It is necessary to note that in the Martio-Rickman-Väisälä theorem, as well as in the Näkki-Palka theorem, the transition to inverse mappings is possible. Of course, we are talking about the situation of homeomorphisms in the first theorem. Based on the analytical definition of quasiconformality, we may conclude that the inverse of a quasiconformal mapping is also quasiconformal, see [ $\mathrm{Va}_{1}$, Theorem 34.3], and thus the aforementioned results can be applied to inverse mappings as well.

The situation will change significantly if a family of mappings with an unbounded characteristic is considered. As we will see from the examples at the end of the article, there are families of maps with unbounded characteristic that are not equicontinuous, however, the inverses to them are such. A similar remark applies to the case related to the equicontinuity of families of maps in the closure of a domain. The problem of equicontinuity for inverse mappings has been considered in [Sev] and [SevSkv] under more restricted conditions than here.

Now we formulate the main assumptions and results. In what follows, $M$ denotes the $n$-modulus of a family of paths, and the element $d m(x)$ corresponds to a Lebesgue measure in $\mathbf{R}^{n}, n \geqslant 2$, see $\left[\mathrm{Va}_{1}\right]$. For the sets $A, B \subset \mathbf{R}^{n}$ we set, as usual,

$$
\operatorname{diam} A=\sup _{x, y \in A}|x-y|, \quad \operatorname{dist}(A, B)=\inf _{x \in A, y \in B}|x-y| .
$$

For given sets $E$ and $F$ and a given set $A$ in $\overline{\mathbf{R}^{n}}=\mathbf{R}^{n} \cup\{\infty\}$, we denote by $\Gamma(E, F, A)$ the family of all paths $\gamma:[0,1] \rightarrow \overline{\mathbf{R}^{n}}$ joining $E$ and $F$ in $A$, that is, $\gamma(0) \in E$, $\gamma(1) \in F$ and $\gamma(t) \in A$ for all $t \in[0,1]$. Everywhere below, unless otherwise stated, the boundary and the closure of a set are understood in the sense of an extended Euclidean space $\overline{\mathbf{R}^{n}}$. Let $x_{0} \in \bar{D}, x_{0} \neq \infty$,

$$
\begin{aligned}
S\left(x_{0}, r\right) & =\left\{x \in \mathbf{R}^{n}:\left|x-x_{0}\right|=r\right\}, \quad S_{i}=S\left(x_{0}, r_{i}\right), \quad i=1,2, \\
A & =A\left(x_{0}, r_{1}, r_{2}\right)=\left\{x \in \mathbf{R}^{n}: r_{1}<\left|x-x_{0}\right|<r_{2}\right\} .
\end{aligned}
$$

Let $Q: \mathbf{R}^{n} \rightarrow \mathbf{R}^{n}$ be a Lebesgue measurable function satisfying the condition $Q(x) \equiv$ 0 for $x \in \mathbf{R}^{n} \backslash D$. The mapping $f: D \rightarrow \overline{\mathbf{R}^{n}}$ is called a ring $Q$-mapping at the point $x_{0} \in \bar{D} \backslash\{\infty\}$, if the condition

$$
M\left(f\left(\Gamma\left(S_{1}, S_{2}, D\right)\right)\right) \leqslant \int_{A \cap D} Q(x) \cdot \eta^{n}\left(\left|x-x_{0}\right|\right) d m(x)
$$

holds for all $0<r_{1}<r_{2}<d_{0}:=\sup _{x \in D}\left|x-x_{0}\right|$ and all Lebesgue measurable functions $\eta:\left(r_{1}, r_{2}\right) \rightarrow[0, \infty]$ such that

$$
\int_{r_{1}}^{r_{2}} \eta(r) d r \geqslant 1
$$

The mapping of $f$ is called a ring $Q$-mapping in $D$, if condition (1.1) is satisfied at every point $x_{0} \in D$, and a ring $Q$-mapping in $\bar{D}$, if the condition (1.1) holds at every point $x_{0} \in \bar{D}$. For the properties of such mappings see [RSY] and [MRSY $\mathrm{M}_{2}$.

Estimates of the form (1.1) have a large range of applications and inequalities of this type go back to $\left[\mathrm{Va}_{2}\right]$ for quasiconformal and to [Pol] for quasiregular mappings. Inequality (1.1) can be also used for mappings with unbounded characteristics, see [MRSY ${ }_{1}$, Theorems 4.6 and 6.10] and [KO, Theorem 4.1].

A domain $D \subset \mathbf{R}^{n}$ is called locally connected at the point $x_{0}$, if for any neighborhood $U$ of point $x_{0}$ there is a neighborhood $V \subset U$ of the same point such that $V \cap D$ is connected. The domain $D$ is called locally connected on $\partial D$, if this domain is such at each point of its boundary. The boundary of the domain $D$ is called weakly flat at 
the point $x_{0}$, if for every number $P>0$ and for every neighborhood $U$ of this point there is a neighborhood $V$ of point $x_{0}$ such that $M(\Gamma(E, F, D))>P$ for arbitrary continua $E$ and $F$, satisfying conditions $F \cap \partial U \neq \varnothing \neq F \cap \partial V$. The boundary of domain $D$ is called weakly flat if it is such at each point of its boundary.

Let $(X, d)$ and $\left(X^{\prime}, d^{\prime}\right)$ be metric spaces with distances $d$ and $d^{\prime}$, respectively. A family $\mathfrak{G}$ of mappings $g: X^{\prime} \rightarrow X$ is said to be equicontinuous at a point $y_{0} \in X^{\prime}$, if for every $\varepsilon>0$ there is $\delta=\delta\left(\varepsilon, y_{0}\right)>0$ such that $d\left(g(y), g\left(y_{0}\right)\right)<\varepsilon$ for all $g \in \mathfrak{G}$ and $y \in X^{\prime}$ with $d^{\prime}\left(y, y_{0}\right)<\delta$. The family $\mathfrak{G}$ is equicontinuous if $\mathfrak{G}$ is equicontinuous at every point $y_{0} \in X^{\prime}$. In what follows, unless otherwise stated, $X=D$ and $d(x, y)=|x-y|$, where $D$ is a bounded domain of the Euclidean space $\mathbf{R}^{n}$. The space $X^{\prime}$ means either $D^{\prime}$ or $\overline{D^{\prime}}$ depending on the context. In this case, $D^{\prime}$ is the domain of the extended Euclidean space $\overline{\mathbf{R}^{n}}$, and $h$ denotes the so-called chordal metric defined by the equalities

$$
h(x, y)=\frac{|x-y|}{\sqrt{1+|x|^{2}} \sqrt{1+|y|^{2}}}, \quad x \neq \infty \neq y, \quad h(x, \infty)=\frac{1}{\sqrt{1+|x|^{2}}} .
$$

For a given set $E \subset \overline{\mathbf{R}^{n}}$, we set

$$
h(E):=\sup _{x, y \in E} h(x, y) .
$$

The quantity $h(E)$ in (1.4) is called the chordal diameter of the set $E$.

For given domains $D$ and $D^{\prime}$ in $\overline{\mathbf{R}^{n}}$ and given Lebesgue measurable function $Q: \mathbf{R}^{n} \rightarrow[0, \infty]$, which vanishes outside $D$, denote by $\mathfrak{R}_{Q}\left(D, D^{\prime}\right)$ the family of all homeomorphisms $g$ of $D^{\prime}$ onto $D$ such that the mapping $f=g^{-1}$ is $\operatorname{ring} Q$ homeomorphism in $D$. The following result holds.

Theorem 1.5. Let $n \geqslant 2$, and let $D$ be a bounded domain in $\mathbf{R}^{n}$. If $Q \in L^{1}(D)$, then the family $\mathfrak{R}_{Q}\left(D, D^{\prime}\right)$ is equicontinuous in $D^{\prime}$.

Note that Theorem 1.5 does not contain any geometric conditions on $D$ and $D^{\prime}$ except that $D$ is bounded. We also present an equicontinuity result in the closure of a domain. For this we make the following assumptions. Let $D$ and $D^{\prime}$ be domains in $\overline{\mathbf{R}^{n}}, A \subset D$ a continuum and $Q$ a Lebesgue measurable function which vanishes outside $D$. We let $\mathfrak{S}_{\delta, A, Q}\left(D, D^{\prime}\right)$ be the family of all homeomorphisms $g$ of $D^{\prime}$ onto $D$ such that $f=g^{-1}$ is a ring $Q$-homeomorphism in $\bar{D}$, and the condition

$$
h(f(A)):=\sup _{x, y \in f(A)} h(x, y) \geqslant \delta
$$

is fulfilled. The following assertion holds.

Theorem 1.6. Let $n \geqslant 2$, and let $D$ be a bounded domain in $\mathbf{R}^{n}$ which is locally connected at all its boundary points. Suppose that the boundary of the domain $D^{\prime}$ is weakly flat; moreover, no component of $\partial D^{\prime}$ degenerates into a point. If $Q \in L^{1}(D)$, then each map $g \in \mathfrak{S}_{\delta, A, Q}\left(D, D^{\prime}\right)$ has a continuous extension $\bar{g}: \overline{D^{\prime}} \rightarrow \bar{D}$, such that $\bar{g}\left(\overline{D^{\prime}}\right)=\bar{D}$, while the family $\mathfrak{S}_{\delta, A, Q}\left(\bar{D}, \overline{D^{\prime}}\right)$, consisting of all extended mappings, is equicontinuous in $\overline{D^{\prime}}$.

It is quite natural that in Theorem 1.6 there are more conditions than in Theorem 1.5. Indeed, in order for the family of mappings to be equicontinuous in the closure of an domain, at least a continuous extension to its boundary is required. However, even for conformal mappings of the unit disk, this property can be violated if the boundary of the image domain is "too bad". 


\section{Preliminaries}

We need the following topological lemma and refer to [SevSkv] for the proof. Recall that a path will be called a continuous mapping $\gamma: I \rightarrow \mathbf{R}^{n}$ of a segment, interval or half-interval $I \subset \mathbf{R}$ into $n$-dimensional Euclidean space $\mathbf{R}^{n}$. As usual, the following set is called the locus of a path $\gamma: I \rightarrow \mathbf{R}^{n}$ :

$$
|\gamma|=\left\{x \in \mathbf{R}^{n}: \exists t \in I: \gamma(t)=x\right\}
$$

We also say that the paths $\gamma_{1}$ and $\gamma_{2}$ do not intersect each other if their loci do not intersect as sets in $\mathbf{R}^{n}$. A path $\gamma: I \rightarrow \mathbf{R}^{n}$ is called a Jordan arc, if $\gamma$ is a homeomorphism of $I$ onto $|\gamma|$.

Lemma 2.1. Let $n \geqslant 2$, and let $D$ be a domain in $\mathbf{R}^{n}$ that is locally connected on its boundary. Then every two pairs of points $a \in D, b \in \bar{D}$ and $c \in D, d \in \bar{D}$ such that $a \neq c$ and $b \neq d$ can be joined by non-intersecting paths $\gamma_{1}:[0,1] \rightarrow \bar{D}$ and $\gamma_{2}:[0,1] \rightarrow \bar{D}$ so that $\gamma_{i}(t) \in D$ for all $t \in(0,1)$ and all $i=1,2$, while $\gamma_{1}(0)=a$, $\gamma_{1}(1)=b, \gamma_{2}(0)=c$ and $\gamma_{2}(1)=d$.

The following statement is a simple consequence of the well-known Väisälä theorem on the lower estimate of the modulus of families of paths joining two continua that intersect the plates of a spherical ring.

Lemma 2.2. (Väisälä's lemma on the weak flatness of inner points) Let $n \geqslant$ 2 , let $D$ be a domain in $\overline{\mathbf{R}^{n}}$, and let $x_{0} \in D$. Then for each $P>0$ and each neighborhood $U$ of point $x_{0}$ there is a neighborhood $V \subset U$ of the same point such that $M(\Gamma(E, F, D))>P$ for any continua $E, F \subset D$ intersecting $\partial U$ and $\partial V$.

Proof. Fix a neighborhood $U$ of a point $x_{0}$. Without loss of generality, using auxiliary inversion $\varphi(x)=x /|x|^{2}$, if necessary, we may assume that $x_{0} \neq \infty$. Choose now $\varepsilon_{0}>0$ so that $\overline{B\left(x_{0}, \varepsilon_{0}\right)} \subset D \cap U$. Let $c_{n}$ be a positive Väisälä constant defined in $\left[\mathrm{Va}_{1},(10.11)\right]$, and let $\varepsilon \in\left(0, \varepsilon_{0}\right)$ be so small that $c_{n} \cdot \log \frac{\varepsilon_{0}}{\varepsilon}>P$. Let $V:=B\left(x_{0}, \varepsilon\right)$, and let $E, F$ be arbitrary continua intersecting $\partial U$ and $\partial V$. Then also $E$ and $F$ intersect $S\left(x_{0}, \varepsilon_{0}\right)$ and $\partial V$, see [Ku, Theorem 1.I.5.46]. Therefore, the desired conclusion follows from [ $\mathrm{Va}_{1}$, Section 10.12], because

$$
M(\Gamma(E, F, D)) \geqslant c_{n} \cdot \log \frac{\varepsilon_{0}}{\varepsilon}>P .
$$

\section{Proof of Theorem $\mathbf{1 . 5}$}

We prove the theorem 1.5 by contradiction. Suppose that the conclusion of this theorem does not hold, that is, the family of maps $\mathfrak{R}_{Q}\left(D, D^{\prime}\right)$ is not equicontinuous at some point $y_{0} \in D^{\prime}$. Then there is $y_{0} \in D^{\prime}$ with the following property: for each $m \in \mathbf{N}$ there are $y_{m} \in D^{\prime}$ and a homeomorphism $g_{m} \in \mathfrak{R}_{Q}\left(D, D^{\prime}\right)$, such that $h\left(y_{m}, y_{0}\right)<1 / m$, however,

$$
\left|g_{m}\left(y_{m}\right)-g_{m}\left(y_{0}\right)\right| \geqslant \varepsilon_{0}
$$

Consider a straight line

$$
r=r_{m}(t)=g_{m}\left(y_{0}\right)+\left(g_{m}\left(y_{m}\right)-g_{m}\left(y_{0}\right)\right) t, \quad-\infty<t<\infty,
$$

passing through points $g_{m}\left(y_{m}\right)$ and $g_{m}\left(y_{0}\right)$, see Figure 1 . 


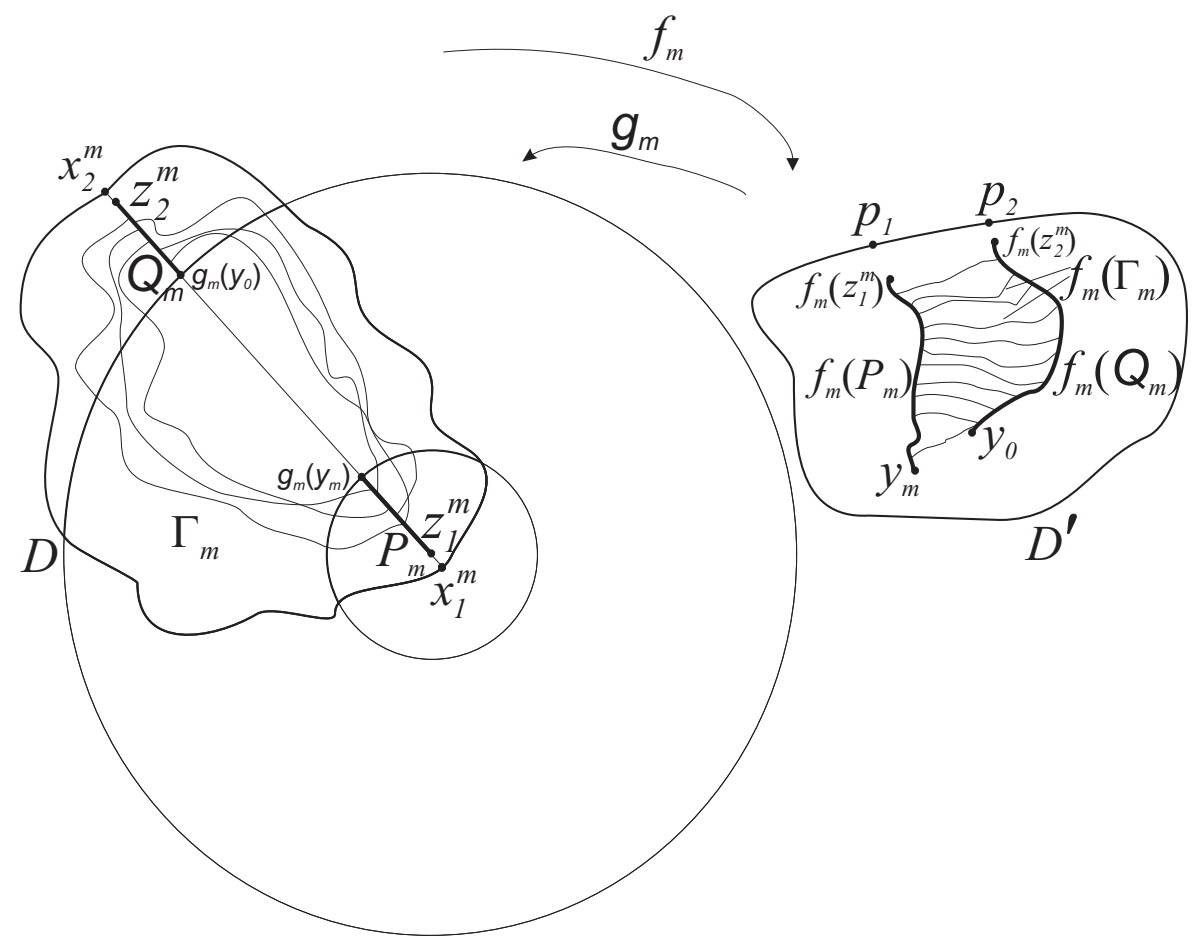

Figure 1. To the proof of the theorem 1.5.

Since the domain $D$ is bounded, in view of [Ku, Theorem 1.I.5.46] the above line $r=r_{m}(t)$ intersects $\partial D$ for some value of the parameter $t \geqslant 1$. In this case, there is $t_{1}^{m} \geqslant 1$ such that $r_{m}\left(t_{1}^{m}\right)=x_{1}^{m} \in \partial D$. Without loss of generality, we may assume that $r_{m}(t) \in D$ for all $t \in\left[1, t_{1}^{m}\right)$. In this case, the segment $\gamma_{1}^{m}(t)=$ $g_{m}\left(y_{0}\right)+\left(g_{m}\left(y_{m}\right)-g_{m}\left(y_{0}\right)\right) t, t \in\left[1, t_{1}^{m}\right]$, belongs to the domain $D$ for all $t \in\left[1, t_{1}^{m}\right)$, $\gamma_{1}^{m}\left(t_{1}^{m}\right)=x_{1}^{m} \in \partial D$ and $\gamma_{1}^{m}(1)=g_{m}\left(y_{m}\right)$. Similarly, there are $t_{2}^{m}<0$ and a segment $\gamma_{2}^{m}(t)=g_{m}\left(y_{0}\right)+\left(g_{m}\left(y_{m}\right)-g_{m}\left(y_{0}\right)\right) t, t \in\left[t_{2}^{m}, 0\right]$, such that $\gamma_{2}^{m}\left(t_{2}^{m}\right)=x_{2}^{m} \in \partial D$, $\gamma_{2}^{m}(0)=g_{m}\left(y_{0}\right)$ and $\gamma_{2}^{m}(t) \in D$ for all $t \in\left(t_{2}^{m}, 0\right]$. Set $f_{m}:=g_{m}^{-1}$ and fix $m \in \mathbf{N}$. Since $f_{m}$ is a homeomorphism, the limit sets $C\left(f_{m}, x_{1}^{m}\right)$ and $C\left(f_{m}, x_{2}^{m}\right)$ are in the set $\partial D^{\prime}$ (see $\left[\mathrm{MRSY}_{3}\right.$, Proposition 13.5]), where, as usual, we use the notation

$$
C(f, x):=\left\{y \in \overline{\mathbf{R}^{n}}: \exists x_{k} \in D: x_{k} \rightarrow x, f\left(x_{k}\right) \rightarrow y, k \rightarrow \infty\right\} .
$$

Therefore, there is a point $z_{1}^{m} \in D \cap\left|\gamma_{1}^{m}\right|$ such that $h\left(f_{m}\left(z_{1}^{m}\right), \partial D^{\prime}\right)<1 / m$. Since the space $\overline{\mathbf{R}^{n}}$ is compact, we may assume that $f_{m}\left(z_{1}^{m}\right) \rightarrow p_{1} \in \partial D^{\prime}$ as $m \rightarrow \infty$. Using similar reasoning, we also conclude that there is a sequence $z_{2}^{m} \in D \cap\left|\gamma_{2}^{m}\right|$ such that $h\left(f_{m}\left(z_{2}^{m}\right), \partial D^{\prime}\right)<1 / m$ and $f_{m}\left(z_{2}^{m}\right) \rightarrow p_{2} \in \partial D^{\prime}$ as $m \rightarrow \infty$. Denote by $P_{m}$ the part of the segment $\gamma_{1}^{m}$, located between points $g_{m}\left(y_{m}\right)$ and $z_{1}^{m}$, and by $Q_{m}$ the corresponding part of the segment $\gamma_{2}^{m}$, located between the points $g_{m}\left(y_{0}\right)$ and $z_{2}^{m}$. Put

where

$$
A_{m}:=A\left(z_{1}^{m}, \varepsilon_{1}^{m}, \varepsilon_{2}^{m}\right)=\left\{x \in \mathbf{R}^{n}: \varepsilon_{1}^{m}<\left|x-z_{1}^{m}\right|<\varepsilon_{2}^{m}\right\}
$$

$$
\varepsilon_{1}^{m}:=\left|g_{m}\left(y_{m}\right)-z_{1}^{m}\right|, \varepsilon_{2}^{m}:=\left|g_{m}\left(y_{0}\right)-z_{1}^{m}\right| .
$$

Let $\Gamma_{m}=\Gamma\left(P_{m}, Q_{m}, D\right)$. Let us show that

$$
\Gamma_{m}>\Gamma\left(S\left(z_{1}^{m}, \varepsilon_{1}^{m}\right), S\left(z_{1}^{m}, \varepsilon_{2}^{m}\right), A_{m} \cap D\right) .
$$

Indeed, let $\gamma \in \Gamma_{m}$, in other words, $\gamma=\gamma(s):[0,1] \rightarrow \mathbf{R}^{n}, \gamma(0) \in P_{m}, \gamma(1) \in Q_{m}$ and $\gamma(s) \in D$ for $0<s<1$. Let $q_{m}>1$ be a number for which

$$
z_{1}^{m}=g_{m}\left(y_{0}\right)+\left(g_{m}\left(y_{m}\right)-g_{m}\left(y_{0}\right)\right) q_{m} \text {. }
$$


Since $\gamma(0) \in P_{m}$, there is $1 \leqslant t_{m} \leqslant q_{m}$ such that

$$
\gamma(0)=g_{m}\left(y_{0}\right)+\left(g_{m}\left(y_{m}\right)-g_{m}\left(y_{0}\right)\right) t_{m} .
$$

Therefore,

$$
\begin{aligned}
\left|\gamma(0)-z_{1}^{m}\right| & =\left|\left(g_{m}\left(y_{m}\right)-g_{m}\left(y_{0}\right)\right)\left(q_{m}-t_{m}\right)\right| \\
& \leq\left|\left(g_{m}\left(y_{m}\right)-g_{m}\left(y_{0}\right)\right)\left(q_{m}-1\right)\right| \\
& \left.=\mid\left(g_{m}\left(y_{m}\right)-g_{m}\left(y_{0}\right)\right) q_{m}+g_{m}\left(y_{0}\right)-g_{m}\left(y_{m}\right)\right) \mid \\
& =\left|g_{m}\left(y_{m}\right)-z_{1}^{m}\right|=\varepsilon_{1}^{m} .
\end{aligned}
$$

On the other hand, since $\gamma(1) \in Q_{m}$, then there exists $p_{m} \leqslant 0$ such that

$$
\gamma(1)=g_{m}\left(y_{0}\right)+\left(g_{m}\left(y_{m}\right)-g_{m}\left(y_{0}\right)\right) p_{m} .
$$

In this case, we obtain that

$$
\begin{aligned}
\left|\gamma(1)-z_{1}^{m}\right| & =\left|\left(g_{m}\left(y_{m}\right)-g_{m}\left(y_{0}\right)\right)\left(q_{m}-p_{m}\right)\right| \\
& \geq\left|\left(g_{m}\left(y_{m}\right)-g_{m}\left(y_{0}\right)\right) q_{m}\right| \\
& =\left|\left(g_{m}\left(y_{m}\right)-g_{m}\left(y_{0}\right)\right) q_{m}+g_{m}\left(y_{0}\right)-g_{m}\left(y_{0}\right)\right| \\
& =\left|g_{m}\left(y_{0}\right)-z_{1}^{m}\right|=\varepsilon_{2}^{m} .
\end{aligned}
$$

Note that

$$
\begin{aligned}
\left|g_{m}\left(y_{0}\right)-g_{m}\left(y_{m}\right)\right|+\varepsilon_{1}^{m} & =\left|g_{m}\left(y_{0}\right)-g_{m}\left(y_{m}\right)\right|+\left|g_{m}\left(y_{m}\right)-z_{1}^{m}\right| \\
& =\left|z_{1}^{m}-g_{m}\left(y_{0}\right)\right|=\varepsilon_{2}^{m},
\end{aligned}
$$

and, therefore, $\varepsilon_{1}^{m}<\varepsilon_{2}^{m}$. Then we obtain from relation (3.4) that

$$
\left|\gamma(1)-z_{1}^{m}\right|>\varepsilon_{1}^{m}
$$

If $\gamma(0) \notin S\left(z_{1}^{m}, \varepsilon_{1}^{m}\right)$, then from the relations (3.3) and (3.6) it follows that $|\gamma| \cap$ $B\left(z_{1}^{m}, \varepsilon_{1}^{m}\right) \neq \varnothing \neq\left(D \backslash B\left(z_{1}^{m}, \varepsilon_{1}^{m}\right)\right) \cap|\gamma|$. Then, in view of [Ku, Theorem 1.I.5.46], there is $t_{1} \in(0,1)$ such that $\gamma\left(t_{1}\right) \in S\left(z_{1}^{m}, \varepsilon_{1}^{m}\right)$. Without loss of generality, we may assume that $\gamma(t) \notin B\left(z_{1}^{m}, \varepsilon_{1}^{m}\right)$ for $t \in\left(t_{1}, 1\right)$. Put $\gamma_{1}:=\left.\gamma\right|_{\left[t_{1}, 1\right]}$.

On the other hand, since $\varepsilon_{1}^{m}<\varepsilon_{2}^{m}$ and $\gamma_{1}\left(t_{1}\right) \in S\left(z_{1}^{m}, \varepsilon_{1}^{m}\right)$, we obtain that $\left|\gamma_{1}\right| \cap B\left(z_{1}^{m}, \varepsilon_{2}^{m}\right) \neq \varnothing$. By (3.4), we obtain $\left(D \backslash B\left(z_{1}^{m}, \varepsilon_{2}^{m}\right)\right) \cap\left|\gamma_{1}\right| \neq \varnothing$. Therefore, by $\left[\mathrm{Ku}\right.$, Theorem 1.I.5.46] there is $t_{2} \in\left[t_{1}, 1\right)$ such that $\gamma_{1}\left(t_{2}\right) \in S\left(z_{1}^{m}, \varepsilon_{2}^{m}\right)$.

Without loss of generality, we may assume that $\gamma_{1}(t) \in B\left(z_{1}^{m}, \varepsilon_{2}^{m}\right)$ for $t \in\left(t_{1}, t_{2}\right)$. Put $\gamma_{2}:=\left.\gamma\right|_{\left[t_{1}, t_{2}\right]}$. Then $\gamma>\gamma_{2}$ and $\gamma_{2} \in \Gamma\left(S\left(z_{1}^{m}, \varepsilon_{1}^{m}\right), S\left(z_{1}^{m}, \varepsilon_{2}^{m}\right), A_{m}\right)$. Thus, the relation (3.2) is now established.

Put

$$
\eta(t)= \begin{cases}\frac{1}{\varepsilon_{0}}, & t \in\left[\varepsilon_{1}^{m}, \varepsilon_{2}^{m}\right], \\ 0, & t \notin\left[\varepsilon_{1}^{m}, \varepsilon_{2}^{m}\right] .\end{cases}
$$

Observe that $\eta$ satisfies the relation (1.2) with $r_{1}=\varepsilon_{1}^{m}$ and $r_{2}=\varepsilon_{2}^{m}$. In fact, we obtain from (3.1) and (3.5) that

$$
r_{1}-r_{2}=\varepsilon_{2}^{m}-\varepsilon_{1}^{m}=\left|g_{m}\left(y_{0}\right)-z_{1}^{m}\right|-\left|g_{m}\left(y_{m}\right)-z_{1}^{m}\right|=\left|g_{m}\left(y_{m}\right)-g_{m}\left(y_{0}\right)\right| \geqslant \varepsilon_{0} .
$$

Then $\int_{\varepsilon_{1}^{m}}^{\varepsilon_{2}^{m}} \eta(t) d t=\left(1 / \varepsilon_{0}\right) \cdot\left(\varepsilon_{2}^{m}-\varepsilon_{1}^{m}\right) \geqslant 1$. By the definition of the corresponding class of mappings in (3.2) considered at the point $z_{1}^{m}$, we obtain that

$$
\begin{aligned}
M\left(f_{m}\left(\Gamma_{m}\right)\right) & \leqslant M\left(f_{m}\left(\Gamma\left(S\left(z_{1}^{m}, \varepsilon_{1}^{m}\right), S\left(z_{1}^{m}, \varepsilon_{2}^{m}\right), A_{m} \cap D\right)\right)\right) \\
& \leqslant \frac{1}{\varepsilon_{0}^{n}} \int_{D} Q(x) d m(x):=c<\infty,
\end{aligned}
$$


since $Q \in L^{1}(D)$.

Relation (3.7) is a uniform estimate of the corresponding modulus of families of paths, in which "uniformity" refers to the index $m$ on the left; however, the righthand side of this relation does not depend on $m$. Now we show that this leads to a contradiction with the condition of the weak flatness of the interior point $y_{0}$, in which we consider our family of maps $\mathfrak{R}_{Q}\left(D, D^{\prime}\right)$. Indeed, first of all we have that

$$
h\left(f_{m}\left(P_{m}\right)\right) \geqslant h\left(y_{m}, f_{m}\left(z_{1}^{m}\right)\right) \geqslant(1 / 2) \cdot h\left(y_{0}, p_{1}\right)>0
$$

and

$$
h\left(f_{m}\left(Q_{m}\right)\right) \geqslant h\left(y_{0}, f_{m}\left(z_{2}^{m}\right)\right) \geqslant(1 / 2) \cdot h\left(y_{0}, p_{2}\right)>0
$$

for large $m \in \mathbf{N}$. (Recall that chordal diameter $h\left(f_{m}\left(Q_{m}\right)\right.$ ) of the set $E$ is defined by the relation (1.4), where, in this case, $\left.E:=f_{m}\left(Q_{m}\right)\right)$. Moreover, note that

$$
h\left(f_{m}\left(P_{m}\right), f_{m}\left(Q_{m}\right)\right):=\inf _{x \in f_{m}\left(P_{m}\right), y \in f_{m}\left(Q_{m}\right)} h(x, y) \leqslant h\left(y_{m}, y_{0}\right) \rightarrow 0, \quad m \rightarrow \infty .
$$

Then, by Lemma 2.2, we have that

$$
M\left(f_{m}\left(\Gamma_{m}\right)\right)=M\left(\Gamma\left(f_{m}\left(P_{m}\right), f_{m}\left(Q_{m}\right), D^{\prime}\right)\right) \rightarrow \infty, \quad m \rightarrow \infty,
$$

which contradicts (3.7) and hence also (3.1). The obtained contradiction refutes the assumption made in (3.1). The theorem is proved.

\section{On the behavior of mappings in the closure of an domain}

We now pose the question of the equicontinuity of families of mappings not only at internal, but also boundary points. In order to fully understand this question, we prove one important topological statement about the approach of the image of the continuum to the boundary of the domain. Note that similar statements were previously known for quasiconformal mappings, see, for example, [Va $\mathrm{Va}_{1}$, Theorems 21.13 and 21.14]. However, we get this result for more general classes of mappings and according to our own scheme of arguments, different from $\left[\mathrm{Va}_{1}\right]$. The meaning of this statement is that the image of a fixed continuum under mappings satisfying the estimate (1.1) cannot approach the boundary of the image domain if this domain has some "good" properties and the diameter of the image of the continuum is bounded below.

Lemma 4.1. Let $n \geqslant 2$, let $D$ be a bounded domain in $\mathbf{R}^{n}$, and let $D^{\prime}$ be some domain in $\overline{\mathbf{R}^{n}}$. Suppose that $D$ is locally connected on $\partial D, D^{\prime}$ has a weakly flat boundary, $Q \in L^{1}(D)$ and, moreover, no connected component of the set $\partial D^{\prime}$ does not degenerate into a point. Let $f_{m}: D \rightarrow D^{\prime}$ be a sequence of homeomorphisms of $D$ onto $D^{\prime}$, satisfying the relation (1.1) in $D$ with the same function $Q$. Suppose also that there is a continuum $A \subset D$ and a number $\delta>0$ such that $h\left(f_{m}(A)\right) \geqslant \delta>0$ for all $m=1,2, \ldots$, where, as usual, $h\left(f_{m}(A)\right)$ is defined in (1.4). Then there is $\delta_{1}>0$ such that

$$
\begin{gathered}
\text { where } h\left(f_{m}(A), \partial D^{\prime}\right)=\inf _{x \in f_{m}(A), y \in \partial D^{\prime}} h(x, y) .
\end{gathered}
$$

Proof. Since $D$ is a bounded domain and, moreover, $f_{m}(D)=D^{\prime}, m=1,2, \ldots$, then $\partial D^{\prime} \neq \varnothing$. Thus, the distance $h\left(f_{m}(A), \partial D^{\prime}\right)$ is well defined.

We carry out the proof by contradiction. Suppose that the conclusion of the lemma is not true. Then for each $k \in \mathbf{N}$ there is some number $m=m_{k}$ such that $h\left(f_{m_{k}}(A), \partial D^{\prime}\right)<1 / k$. Of course, we can assume that the sequence $m_{k}$ increases on 
$k$. Since $\overline{\mathbf{R}^{n}}$ is compact, the set $\partial D^{\prime}$ is also compact in extended Euclidean space. Note that the set $f_{m_{k}}(A)$ is compact as a continuous image of a compact set $A \subset D$ under the mapping $f_{m_{k}}$. In this case, there are elements $x_{k} \in f_{m_{k}}(A)$ and $y_{k} \in \partial D^{\prime}$ such that $h\left(f_{m_{k}}(A), \partial D^{\prime}\right)=h\left(x_{k}, y_{k}\right)<1 / k$ (see Figure 2$)$.

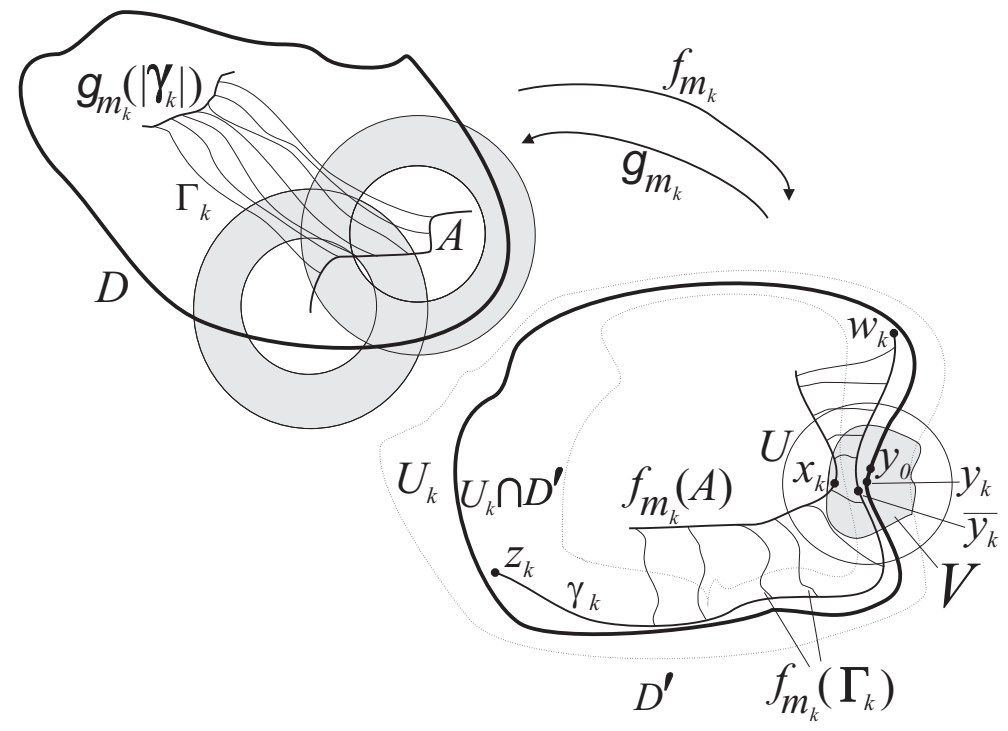

Figure 2. To the proof of Lemma 4.1.

Since $\partial D^{\prime}$ is a compact set, we may assume that $y_{k} \rightarrow y_{0} \in \partial D^{\prime}$ as $k \rightarrow \infty$; then also

$$
x_{k} \rightarrow y_{0} \in \partial D^{\prime}, \quad k \rightarrow \infty .
$$

Let $K_{0}$ be a connected component of the set $\partial D^{\prime}$, containing $y_{0}$. Obviously, $K_{0}$ is a continuum in $\overline{\mathbf{R}^{n}}$. Since $D^{\prime}$ has a weakly flat boundary, the mapping $g_{m_{k}}:=f_{m_{k}}^{-1}$ can be extended to a continuous mapping $\bar{g}_{m_{k}}: \overline{D^{\prime}} \rightarrow \bar{D}$ (see [Sm, Theorem 3]). Moreover, $\bar{g}_{m_{k}}$ is uniformly continuous on the set $\overline{D^{\prime}}$ for every fixed $k$, because the mapping $\bar{g}_{m_{k}}$ is continuous on the compact set $\overline{D^{\prime}}$. In this case, for each $\varepsilon>0$ there is $\delta_{k}=\delta_{k}(\varepsilon)<1 / k$ such that

$$
\left|\bar{g}_{m_{k}}(x)-\bar{g}_{m_{k}}\left(x_{0}\right)\right|<\varepsilon \quad \forall x, x_{0} \in \overline{D^{\prime}}, \quad h\left(x, x_{0}\right)<\delta_{k}, \quad \delta_{k}<1 / k .
$$

Choose $\varepsilon>0$ such that

$$
\varepsilon<(1 / 2) \cdot \operatorname{dist}(\partial D, A)
$$

Denote $B_{h}\left(x_{0}, r\right)=\left\{x \in \overline{\mathbf{R}^{n}}: h\left(x, x_{0}\right)<r\right\}$. For a given $k \in \mathbf{N}$, we set

$$
B_{k}:=\bigcup_{x_{0} \in K_{0}} B_{h}\left(x_{0}, \delta_{k}\right), \quad k \in \mathbf{N} .
$$

Since the set $B_{k}$ is a neighborhood of the continuum $K_{0}$, due to [HK, Lemma 2.2] there is a neighborhood $U_{k}$ of the set $K_{0}$, such that $U_{k} \subset B_{k}$ and $U_{k} \cap D^{\prime}$ is connected. Without loss of generality, we may assume that $U_{k}$ is an open set, so $U_{k} \cap D^{\prime}$ is also path connected (see $\left[\mathrm{MRSY}_{3}\right.$, Proposition 13.1]). Let $h\left(K_{0}\right)=m_{0}$. In this case, there are $z_{0}, w_{0} \in K_{0}$ such that $h\left(K_{0}\right)=h\left(z_{0}, w_{0}\right)=m_{0}$. So, there are sequences $\overline{y_{k}} \in U_{k} \cap D^{\prime}, z_{k} \in U_{k} \cap D^{\prime}$ and $w_{k} \in U_{k} \cap D^{\prime}$ such that $z_{k} \rightarrow z_{0}, \overline{y_{k}} \rightarrow y_{0}$ and $w_{k} \rightarrow w_{0}$ as $k \rightarrow \infty$. We may assume that

$$
h\left(z_{k}, w_{k}\right)>m_{0} / 2 \quad \forall k \in \mathbf{N} .
$$


Since the set $U_{k} \cap D^{\prime}$ is path-connected, we can sequentially join the points $z_{k}, \overline{y_{k}}$ and $w_{k}$ using some path $\gamma_{k}$ such that $\left|\gamma_{k}\right| \subset U_{k} \cap D^{\prime}$. As usual, we denote by $\left|\gamma_{k}\right|$ the locus of the path $\gamma_{k}$ in the domain $D^{\prime}$. Then $g_{m_{k}}\left(\left|\gamma_{k}\right|\right)$ is a compact set in the domain $D$. If $x \in\left|\gamma_{k}\right|$, then there is $x_{0} \in K_{0}$ such that $x \in B\left(x_{0}, \delta_{k}\right)$. Put $\omega \in A \subset D$. Since $x \in\left|\gamma_{k}\right|$ and, moreover, $x$ is an inner point of the domain $D^{\prime}$, we can write here $g_{m_{k}}(x)$ instead of $\bar{g}_{m_{k}}(x)$. By the relations (4.2) and (4.3), as well as by the triangle inequality, we obtain that for sufficiently large $k \in \mathbf{N}$,

$$
\begin{aligned}
\left|g_{m_{k}}(x)-\omega\right| & \geqslant\left|\omega-\bar{g}_{m_{k}}\left(x_{0}\right)\right|-\left|\bar{g}_{m_{k}}\left(x_{0}\right)-g_{m_{k}}(x)\right| \\
& \geqslant \operatorname{dist}(\partial D, A)-(1 / 2) \cdot \operatorname{dist}(\partial D, A)=(1 / 2) \cdot \operatorname{dist}(\partial D, A)>\varepsilon
\end{aligned}
$$

where dist $(\partial D, A):=\inf _{x \in \partial D, y \in A}|x-y|$. Taking inf in the relation (4.5) over all $x \in\left|\gamma_{k}\right|$ and $\omega \in A$, we obtain that

$$
\operatorname{dist}\left(g_{m_{k}}\left(\left|\gamma_{k}\right|\right), A\right):=\inf _{x \in g_{m_{k}}\left(\left|\gamma_{k}\right|\right), y \in A}|x-y|>\varepsilon, \quad \forall k=1,2, \ldots
$$

We cover the continuum $A$ with balls $B(x, \varepsilon / 4), x \in A$. Since $A$ is a compact set, we may assume that $A \subset \bigcup_{i=1}^{M_{0}} B\left(x_{i}, \varepsilon / 4\right), x_{i} \in A, i=1,2, \ldots, M_{0}, 1 \leqslant M_{0}<\infty$. By definition, $M_{0}$ depends only on $A$, in particular, $M_{0}$ does non depend on $k$. We set

$$
\Gamma_{k}:=\Gamma\left(A, g_{m_{k}}\left(\left|\gamma_{k}\right|\right), D\right) .
$$

Note that

$$
\Gamma_{k}=\bigcup_{i=1}^{M_{0}} \Gamma_{k i},
$$

where $\Gamma_{k i}$ consists of all paths $\gamma:[0,1] \rightarrow D$, belonging to the family $\Gamma_{k}$, such that $\gamma(0) \in B\left(x_{i}, \varepsilon / 4\right)$ and $\gamma(1) \in g_{m_{k}}\left(\left|\gamma_{k}\right|\right)$. We now show that

$$
\Gamma_{k i}>\Gamma\left(S\left(x_{i}, \varepsilon / 4\right), S\left(x_{i}, \varepsilon / 2\right), A\left(x_{i}, \varepsilon / 4, \varepsilon / 2\right)\right) .
$$

Indeed, let $\gamma \in \Gamma_{k i}$, in other words, $\gamma:[0,1] \rightarrow D, \gamma(0) \in B\left(x_{i}, \varepsilon / 4\right)$ and $\gamma(1) \in$ $g_{m_{k}}\left(\left|\gamma_{k}\right|\right)$. By (4.6), $|\gamma| \cap B\left(x_{i}, \varepsilon / 4\right) \neq \varnothing \neq|\gamma| \cap\left(D \backslash B\left(x_{i}, \varepsilon / 4\right)\right)$. Therefore, by [Ku, Theorem 1.I.5.46] there is $0<t_{1}<1$ with the condition $\gamma\left(t_{1}\right) \in S\left(x_{i}, \varepsilon / 4\right)$. We can assume that $\gamma(t) \notin B\left(x_{i}, \varepsilon / 4\right)$ for $t>t_{1}$. Put $\gamma_{1}:=\left.\gamma\right|_{\left[t_{1}, 1\right]}$. By (4.6), $\left|\gamma_{1}\right| \cap B\left(x_{i}, \varepsilon / 2\right) \neq \varnothing \neq\left|\gamma_{1}\right| \cap\left(D \backslash B\left(x_{i}, \varepsilon / 2\right)\right)$. Thus, by [Ku, Theorem 1.I.5.46] there is $t_{1}<t_{2}<1$ with $\gamma\left(t_{2}\right) \in S\left(x_{i}, \varepsilon / 2\right)$. We may assume that $\gamma(t) \in B\left(x_{i}, \varepsilon / 2\right)$ for $t<t_{2}$. Put $\gamma_{2}:=\left.\gamma\right|_{\left[t_{1}, t_{2}\right]}$. Then, the path $\gamma_{2}$ is a subpath of $\gamma$, which belongs to the family $\Gamma\left(S\left(x_{i}, \varepsilon / 4\right), S\left(x_{i}, \varepsilon / 2\right), A\left(x_{i}, \varepsilon / 4, \varepsilon / 2\right)\right)$. Thus, the relation (4.9) is established. Further reasoning is based, as before, on the successful choice of an admissible function $\eta$. Put

$$
\eta(t)= \begin{cases}4 / \varepsilon, & t \in[\varepsilon / 4, \varepsilon / 2], \\ 0, & t \notin[\varepsilon / 4, \varepsilon / 2] .\end{cases}
$$

Note that $\eta$ satisfies (1.2) for $r_{1}=\varepsilon / 4$ and $r_{2}=\varepsilon / 2$. Then, according to the definition of a ring $Q$-homeomorphism at $x_{i}$, we obtain that

$$
M\left(f_{m_{k}}\left(\Gamma\left(S\left(x_{i}, \varepsilon / 4\right), S\left(x_{i}, \varepsilon / 2\right)\right), A\left(x_{i}, \varepsilon / 4, \varepsilon / 2\right)\right)\right) \leqslant(4 / \varepsilon)^{n} \cdot\|Q\|_{1}<c<\infty,
$$

where $c$ is some positive constant and $\|Q\|_{1}$ is $L_{1}$-norm of the function $Q$ in $D$. By (4.8), (4.9) and (4.10), using the subadditivity of modulus, we obtain that

$$
M\left(f_{m_{k}}\left(\Gamma_{k}\right)\right) \leqslant \frac{4^{n} M_{0}}{\varepsilon^{n}} \int_{D} Q(x) d m(x) \leqslant c \cdot M_{0}<\infty .
$$


The estimate (4.11) contradicts the weak flatness on the boundary of $D^{\prime}$. Indeed, let $P>c \cdot M_{0}$ and $U=B_{h}\left(y_{0}, r_{0}\right)=\left\{y \in \overline{\mathbf{R}^{n}}: h\left(y, y_{0}\right)<r_{0}\right\}$, where $0<r_{0}<$ $\min \left\{\delta / 4, m_{0} / 4\right\}, \delta$ is the number from the condition of the lemma and $h\left(K_{0}\right)=m_{0}$. Note that $\left|\gamma_{k}\right| \cap U \neq \varnothing \neq\left|\gamma_{k}\right| \cap\left(D^{\prime} \backslash U\right)$ for sufficiently large $k \in \mathbf{N}$, since $h\left(\left|\gamma_{k}\right|\right) \geqslant$ $m_{0} / 2>m_{0} / 4, \overline{y_{k}} \in\left|\gamma_{k}\right|$ and $\overline{y_{k}} \rightarrow y_{0}$ as $k \rightarrow \infty$. Similarly, $f_{m_{k}}(A) \cap U \neq \varnothing \neq$ $f_{m_{k}}(A) \cap\left(D^{\prime} \backslash U\right)$. Since $\left|\gamma_{k}\right|$ and $f_{m_{k}}(A)$ are continua, we obtain that

$$
f_{m_{k}}(A) \cap \partial U \neq \varnothing, \quad\left|\gamma_{k}\right| \cap \partial U \neq \varnothing,
$$

see [Ku, Theorem 1.I.5.46]. For a given $P>0$, let $V \subset U$ be a neighborhood of the point $y_{0}$, corresponding to the definition of a weakly flat boundary. Then we have that

$$
M\left(\Gamma\left(E, F, D^{\prime}\right)\right)>P
$$

for any continua $E, F \subset D^{\prime}$ with $E \cap \partial U \neq \varnothing \neq E \cap \partial V$ and $F \cap \partial U \neq \varnothing \neq F \cap \partial V$. Observe that

$$
f_{m_{k}}(A) \cap \partial V \neq \varnothing, \quad\left|\gamma_{k}\right| \cap \partial V \neq \varnothing
$$

for sufficiently large $k \in \mathbf{N}$. Indeed, $\overline{y_{k}} \in\left|\gamma_{k}\right|, x_{k} \in f_{m_{k}}(A)$, where $x_{k}, \overline{y_{k}} \rightarrow y_{0} \in V$ as $k \rightarrow \infty$. Therefore, $\left|\gamma_{k}\right| \cap V \neq \varnothing \neq f_{m_{k}}(A) \cap V$ for large $k \in \mathbf{N}$. In addition, we have that $h(V) \leqslant h(U) \leqslant 2 r_{0}<m_{0} / 2$. By $(4.4), h\left(\left|\gamma_{k}\right|\right)>m_{0} / 2$, therefore, $\left|\gamma_{k}\right| \cap\left(D^{\prime} \backslash V\right) \neq \varnothing$. Thus, by $\left[\mathrm{Ku}\right.$, Theorem 1.I.5.46], $\left|\gamma_{k}\right| \cap \partial V \neq \varnothing$. Similarly, $h(V) \leqslant$ $h(U) \leqslant 2 r_{0}<\delta / 2$. Since $h\left(f_{m_{k}}(A)\right)>\delta$, we obtain that $f_{m_{k}}(A) \cap\left(D^{\prime} \backslash V\right) \neq \varnothing$. By [Ku, Theorem 1.I.5.46], we have that $f_{m_{k}}(A) \cap \partial V \neq \varnothing$. Thus, the relation (4.14) is established.

By (4.12), (4.13) and (4.14), we obtain that

$$
M\left(\Gamma\left(f_{m_{k}}(A),\left|\gamma_{k}\right|, D^{\prime}\right)\right)>P .
$$

Note that $\Gamma\left(f_{m_{k}}(A),\left|\gamma_{k}\right|, D^{\prime}\right)=f_{m_{k}}\left(\Gamma\left(A, g_{m_{k}}\left(\left|\gamma_{k}\right|\right), D\right)\right)=f_{m_{k}}\left(\Gamma_{k}\right)$. Therefore, the relation (4.15) can be written as

$$
M\left(\Gamma\left(f_{m_{k}}(A),\left|\gamma_{k}\right|, D^{\prime}\right)\right)=M\left(f_{m_{k}}\left(\Gamma_{k}\right)\right)>P>c \cdot M_{0} .
$$

In this case, by (4.11) and (4.16), we have that simultaneously $M\left(f_{m_{k}}\left(\Gamma_{k}\right)\right)>c \cdot M_{0}$ and $M\left(f_{m_{k}}\left(\Gamma_{k}\right)\right) \leqslant c \cdot M_{0}$ for sufficiently large $k \in \mathbf{N}$. The resulting contradiction means that the above assumption $h\left(f_{m_{k}}(A), \partial D^{\prime}\right)<1 / k$ was incorrect. The proof of the lemma is complete.

Proof of Theorem 1.6. Let $g \in \mathfrak{S}_{\delta, A, Q}\left(D, D^{\prime}\right)$. Since $D^{\prime}$ has a weakly flat boundary, $g$ can be extended to a continuous mapping $\bar{g}: \overline{D^{\prime}} \rightarrow \bar{D}$ (see [Sm, Theorem 3], see also $\left[\mathrm{MRSY}_{3}\right.$, Theorem 4.6]).

We now verify the equality $\bar{g}\left(\overline{D^{\prime}}\right)=\bar{D}$. Indeed, by definition, $\bar{g}\left(\overline{D^{\prime}}\right) \subset \bar{D}$. It remains to show the converse inclusion $\bar{D} \subset \bar{g}\left(\overline{D^{\prime}}\right)$. Let $x_{0} \in \bar{D}$. Now, we show that $x_{0} \in \bar{g}\left(\overline{D^{\prime}}\right)$. If $x_{0} \in \bar{D}$, then either $x_{0} \in D$, or $x_{0} \in \partial D$. In the case $x_{0} \in D$ there is nothing to prove, since $\bar{g}\left(D^{\prime}\right)=D$ by the condition of the theorem. Now consider the case when $x_{0} \in \partial D$. In this case, there are $x_{k} \in D$ and $y_{k} \in D^{\prime}$ such that $x_{k}=\bar{g}\left(y_{k}\right)$ and $x_{k} \rightarrow x_{0}$ as $k \rightarrow \infty$. Since $\overline{D^{\prime}}$ is a compact set of an extended Euclidean space, we may assume that $y_{k} \rightarrow y_{0} \in \overline{D^{\prime}}$ as $k \rightarrow \infty$. Since $f=g^{-1}$ is a homeomorphism, $y_{0} \in \partial D^{\prime}$. Since the mapping $\bar{g}^{-1}$ is continuous in $\overline{D^{\prime}}$, we have $\bar{g}\left(y_{k}\right) \rightarrow \bar{g}\left(y_{0}\right)$ as $k \rightarrow \infty$. However, in this case, $\bar{g}\left(y_{0}\right)=x_{0}$, because $\bar{g}\left(y_{k}\right)=x_{k}$ and $x_{k} \rightarrow x_{0}$ as $k \rightarrow \infty$. Therefore, $x_{0} \in \bar{g}\left(\overline{D^{\prime}}\right)$. The inclusion $\bar{D} \subset \bar{g}\left(\overline{D^{\prime}}\right)$ is proved. Finally, the relation $\bar{D}=\bar{g}\left(\overline{D^{\prime}}\right)$ is also established, as required. 
The equicontinuity of the family $\mathfrak{S}_{\delta, A, Q}\left(\bar{D}, \overline{D^{\prime}}\right)$ in the domain $D^{\prime}$ is the statement of Theorem 1.5. It remains to establish that the same family of mappings is equicontinuous at the boundary points of the domain $D^{\prime}$.

We carry out the proof by contradiction. Suppose that the above conclusion does not hold. Then there is a point $z_{0} \in \partial D^{\prime}$, a positive number $\varepsilon_{0}>0$, a sequence $z_{m} \in \overline{D^{\prime}}$ converging to a point $z_{0}$ and a map $\bar{g}_{m} \in \mathfrak{S}_{\delta, A, Q}\left(\bar{D}, \overline{D^{\prime}}\right)$ such that

$$
\left|\bar{g}_{m}\left(z_{m}\right)-\bar{g}_{m}\left(z_{0}\right)\right| \geqslant \varepsilon_{0}, \quad m=1,2, \ldots
$$

Put $g_{m}:=\left.\bar{g}_{m}\right|_{D^{\prime}}$. Since the map $g_{m}$ has a continuous extension to $\partial D^{\prime}$, we may assume that $z_{m} \in D^{\prime}$ and, therefore, $\bar{g}_{m}\left(z_{m}\right)=g_{m}\left(z_{m}\right)$. In addition, there is a sequence $z_{m}^{\prime} \in D^{\prime}$ with $z_{m}^{\prime} \rightarrow z_{0}$ as $m \rightarrow \infty$, such that $\left|g_{m}\left(z_{m}^{\prime}\right)-\bar{g}_{m}\left(z_{0}\right)\right| \rightarrow 0$ as $m \rightarrow \infty$. Since the domain $D$ is bounded, $\bar{D}$ is a compact set. Therefore, we may assume that $g_{m}\left(z_{m}\right)$ and $\bar{g}_{m}\left(z_{0}\right)$ converge as $m \rightarrow \infty$. Let $g_{m}\left(z_{m}\right) \rightarrow \overline{x_{1}}$ and $\bar{g}_{m}\left(z_{0}\right) \rightarrow \overline{x_{2}}$ as $m \rightarrow \infty$. By the continuity of the module in (4.17), $\overline{x_{1}} \neq \overline{x_{2}}$. Also, since homeomorphisms preserve the boundary of the domain, $\overline{x_{2}} \in \partial D$. Let $x_{1}$ and $x_{2}$ be different points of the continuum $A$, none of which is the same as $\overline{x_{1}}$. According to Lemma 2.1 we can join pairs of points $x_{1}, \overline{x_{1}}$ and $x_{2}, \overline{x_{2}}$ using the paths $\gamma_{1}:[0,1] \rightarrow \bar{D}$ and $\gamma_{2}:[0,1] \rightarrow \bar{D}$ so that $\left|\gamma_{1}\right| \cap\left|\gamma_{2}\right|=\varnothing, \gamma_{1}(t), \gamma_{2}(t) \in D$ for $t \in(0,1), \gamma_{1}(0)=x_{1}, \gamma_{1}(1)=\overline{x_{1}}, \gamma_{2}(0)=x_{2}$ and $\gamma_{2}(1)=\overline{x_{2}}$. Since the domain $D$ is locally connected on $\partial D$, there are neighborhoods $U_{1}$ and $U_{2}$ of points $\overline{x_{1}}$ and $\overline{x_{2}}$, respectively, whose closures do not intersect, and, moreover, sets $W_{i}:=D \cap U_{i}$ are path-connected. Without loss of generality, we may assume that $\overline{U_{1}} \subset B\left(\overline{x_{1}}, \delta_{0}\right)$ and

$$
\overline{B\left(\overline{x_{1}}, \delta_{0}\right)} \cap\left|\gamma_{2}\right|=\varnothing=\overline{U_{2}} \cap\left|\gamma_{1}\right|, \quad \overline{B\left(\overline{x_{1}}, \delta_{0}\right)} \cap \overline{U_{2}}=\varnothing
$$

In addition, we may assume that $g_{m}\left(z_{m}\right) \in W_{1}$ and $g_{m}\left(z_{m}^{\prime}\right) \in W_{2}$ for all $m \in \mathbf{N}$. Let $a_{1}$ and $a_{2}$ are arbitrary points belonging to $\left|\gamma_{1}\right| \cap W_{1}$ and $\left|\gamma_{2}\right| \cap W_{2}$. Let $0<t_{1}, t_{2}<1$ be such that $\gamma_{1}\left(t_{1}\right)=a_{1}$ and $\gamma_{2}\left(t_{2}\right)=a_{2}$. Join the points $a_{1}$ and $g_{m}\left(z_{m}\right)$ by means of the path $\alpha_{m}:\left[t_{1}, 1\right] \rightarrow W_{1}$ such that $\alpha_{m}\left(t_{1}\right)=a_{1}$ and $\alpha_{m}(1)=g_{m}\left(z_{m}\right)$. Similarly, let us join $a_{2}$ and $g_{m}\left(z_{m}^{\prime}\right)$ by means of the path $\beta_{m}:\left[t_{2}, 1\right] \rightarrow W_{2}, \beta_{m}\left(t_{2}\right)=a_{2}$ and $\beta_{m}(1)=g_{m}\left(z_{m}^{\prime}\right)$ (see Figure 3).

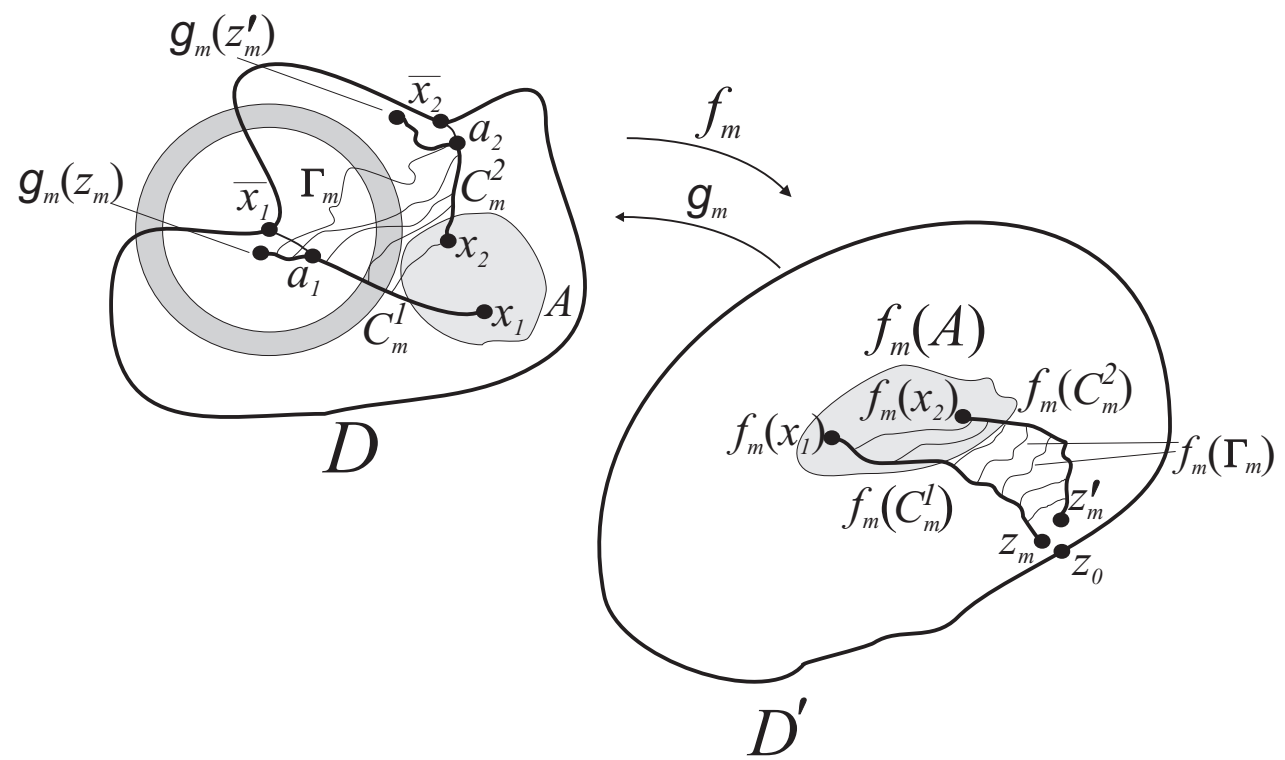

Figure 3. To the proof of Theorem 1.6. 
Put

$$
C_{m}^{1}(t)=\left\{\begin{array}{ll}
\gamma_{1}(t), & t \in\left[0, t_{1}\right], \\
\alpha_{m}(t), & t \in\left[t_{1}, 1\right],
\end{array} \quad C_{m}^{2}(t)= \begin{cases}\gamma_{2}(t), & t \in\left[0, t_{2}\right], \\
\beta_{m}(t), & t \in\left[t_{2}, 1\right] .\end{cases}\right.
$$

As usual, we denote by $\left|C_{m}^{1}\right|$ and $\left|C_{m}^{2}\right|$ the loci of the paths $C_{m}^{1}$ and $C_{m}^{2}$, respectively. Setting

$$
l_{0}=\min \left\{\operatorname{dist}\left(\left|\gamma_{1}\right|,\left|\gamma_{2}\right|\right), \operatorname{dist}\left(\left|\gamma_{1}\right|, U_{2}\right)\right\},
$$

consider the coverage $A_{0}:=\bigcup_{x \in\left|\gamma_{1}\right|} B\left(x, l_{0} / 4\right)$ by balls of $\left|\gamma_{1}\right|$. Since $\left|\gamma_{1}\right|$ is a compact set, we can choose a finite number of indices $1 \leqslant N_{0}<\infty$ and the corresponding points $x_{1}, \ldots, x_{N_{0}} \in\left|\gamma_{1}\right|$ such that $\left|\gamma_{1}\right| \subset B_{0}:=\bigcup_{i=1}^{N_{0}} B\left(x_{i}, l_{0} / 4\right)$. In this case,

$$
\left|C_{m}^{1}\right| \subset U_{1} \cup\left|\gamma_{1}\right| \subset \overline{B\left(\overline{x_{1}}, \delta_{0}\right)} \cup \bigcup_{i=1}^{N_{0}} B\left(x_{i}, l_{0} / 4\right) .
$$

Let $\Gamma_{m}$ be a family of paths joining the sets $\left|C_{m}^{1}\right|$ and $\left|C_{m}^{2}\right|$ in the domain $D$. Then we will have that

$$
\Gamma_{m}=\bigcup_{i=0}^{N_{0}} \Gamma_{m i}
$$

where $\Gamma_{m i}$ is a family consisting of paths $\gamma:[0,1] \rightarrow D$ such that $\gamma(0) \in B\left(x_{i}, l_{0} / 4\right) \cap$ $\left|C_{m}^{1}\right|$ and $\gamma(1) \in\left|C_{2}^{m}\right|$ at $1 \leqslant i \leqslant N_{0}$. Similarly, define $\Gamma_{m 0}$ as a family, consisting of paths $\gamma:[0,1] \rightarrow D$ such that $\gamma(0) \in B\left(\overline{x_{1}}, \delta_{0}\right) \cap\left|C_{m}^{1}\right|$ and $\gamma(1) \in\left|C_{2}^{m}\right|$. By (4.18) there is $\sigma_{0}>\delta_{0}>0$ such that

$$
\overline{B\left(\overline{x_{1}}, \sigma_{0}\right)} \cap\left|\gamma_{2}\right|=\varnothing=\overline{U_{2}} \cap\left|\gamma_{1}\right|, \quad \overline{B\left(\overline{x_{1}}, \sigma_{0}\right)} \cap \overline{U_{2}}=\varnothing .
$$

Arguing as in the proof of Lemma 4.1, we may show that

$$
\begin{aligned}
\Gamma_{m 0} & >\Gamma\left(S\left(\overline{x_{1}}, \delta_{0}\right), S\left(\overline{x_{1}}, \sigma_{0}\right), A\left(\overline{x_{1}}, \delta_{0}, \sigma_{0}\right) \cap D\right), \\
\Gamma_{m i} & >\Gamma\left(S\left(x_{i}, l_{0} / 4\right), S\left(x_{i}, l_{0} / 2\right), A\left(x_{i}, l_{0} / 4, l_{0} / 2\right) \cap D\right) .
\end{aligned}
$$

Put

$$
\eta(t)=\left\{\begin{array}{ll}
4 / l_{0}, & t \in\left[l_{0} / 4, l_{0} / 2\right], \\
0, & t \notin\left[l_{0} / 4, l_{0} / 2\right],
\end{array} \quad \eta_{0}(t)= \begin{cases}1 /\left(\sigma_{0}-\delta_{0}\right), & t \in\left[\delta_{0}, \sigma_{0}\right], \\
0, & t \notin\left[\delta_{0}, \sigma_{0}\right] .\end{cases}\right.
$$

Let $f_{m}:=g_{m}^{-1}$. Then by virtue of (1.1), we obtain that

$$
\begin{aligned}
M\left(f_{m}\left(\Gamma\left(S\left(\overline{x_{1}}, \delta_{0}\right), S\left(\overline{x_{1}}, \sigma_{0}\right), A\left(\overline{x_{1}}, \delta_{0}, \sigma_{0}\right) \cap D\right)\right)\right) & \leqslant\left(1 /\left(\sigma_{0}-\delta_{0}\right)\right)^{n} \cdot\|Q\|_{1} \\
& <c_{1}<\infty, \\
M\left(f_{m}\left(\Gamma\left(S\left(x_{i}, l_{0} / 4\right), S\left(x_{i}, l_{0} / 2\right), A\left(x_{i}, l_{0} / 4, l_{0} / 2\right) \cap D\right)\right)\right) & \leqslant\left(4 /\left(l_{0}\right)\right)^{n} \cdot\|Q\|_{1} \\
& <c_{2}<\infty,
\end{aligned}
$$

where $c_{1}$ and $c_{1}$ are some positive constants independent on $m$. Combining relations (4.19), (4.20) and (4.21) and taking into account the subadditivity of the modulus of families of paths, we obtain that

$$
M\left(f_{m}\left(\Gamma_{m}\right)\right) \leqslant\left(4^{n} N_{0} / l_{0}^{n}+\left(1 /\left(\sigma_{0}-\delta_{0}\right)\right)^{n}\right)\|Q\|_{1}:=c<\infty .
$$

Again, as in the proof of Lemma 4.1, we show that relation (4.22) contradicts the condition of the weak plane of the mapped domain. Indeed, by Lemma 4.1, there is 
a number $\delta_{1}>0$ such that $h\left(f_{m}(A), \partial D^{\prime}\right)>\delta_{1}>0$ for all $m=1,2, \ldots$ Therefore,

$$
\begin{gathered}
h\left(\left|f_{m}\left(C_{m}^{1}\right)\right|\right) \geqslant h\left(z_{m}, f_{m}\left(x_{1}\right)\right) \geqslant(1 / 2) \cdot h\left(f_{m}(A), \partial D^{\prime}\right)>\delta_{1} / 2, \\
h\left(\left|f_{m}\left(C_{m}^{2}\right)\right|\right) \geqslant h\left(z_{m}^{\prime}, f_{m}\left(x_{2}\right)\right) \geqslant(1 / 2) \cdot h\left(f_{m}(A), \partial D^{\prime}\right)>\delta_{1} / 2
\end{gathered}
$$

for some $M_{0} \in \mathbf{N}$ and all $m \geqslant M_{0}$. Put $U:=B_{h}\left(z_{0}, r_{0}\right)=\left\{y \in \overline{\mathbf{R}^{n}}: h\left(y, z_{0}\right)<\right.$ $\left.r_{0}\right\}$, where $0<r_{0}<\delta_{1} / 4$ and the number $\delta_{1}$ is from the relation (4.23). Note that $\left|f_{m}\left(C_{m}^{1}\right)\right| \cap U \neq \varnothing \neq\left|f_{m}\left(C_{m}^{1}\right)\right| \cap\left(D^{\prime} \backslash U\right)$ for sufficiently large $m \in \mathbf{N}$, since $h\left(\left|f_{m}\left(C_{m}^{1}\right)\right|\right) \geqslant \delta_{1} / 2$ and $z_{m} \in\left|f_{m}\left(C_{m}^{1}\right)\right|, z_{m} \rightarrow z_{0}$ as $m \rightarrow \infty$. Similarly, $\left|f_{m}\left(C_{m}^{2}\right)\right| \cap$ $U \neq \varnothing \neq\left|f_{m}\left(C_{m}^{2}\right)\right| \cap\left(D^{\prime} \backslash U\right)$. Since $\left|f_{m}\left(C_{m}^{1}\right)\right|$ and $\left|f_{m}\left(C_{m}^{2}\right)\right|$ are continua,

$$
\left|f_{m}\left(C_{m}^{1}\right)\right| \cap \partial U \neq \varnothing, \quad\left|f_{m}\left(C_{m}^{2}\right)\right| \cap \partial U \neq \varnothing,
$$

see, e.g., $\left[\mathrm{Ku}\right.$, Theorem 1.I.5.46]. Since $\partial D^{\prime}$ is weakly flat, then for a given $P>0$ there is a neighborhood $V \subset U$ of $z_{0}$ such that

$$
M\left(\Gamma\left(E, F, D^{\prime}\right)\right)>P
$$

for any continua $E, F \subset D^{\prime}$ with $E \cap \partial U \neq \varnothing \neq E \cap \partial V$ and $F \cap \partial U \neq \varnothing \neq F \cap \partial V$. We show that the following relation holds:

$$
\left|f_{m}\left(C_{m}^{1}\right)\right| \cap \partial V \neq \varnothing, \quad\left|f_{m}\left(C_{m}^{2}\right)\right| \cap \partial V \neq \varnothing
$$

where $m \in \mathbf{N}$ is large enough.

Indeed, let $z_{m} \in\left|f_{m}\left(C_{m}^{1}\right)\right|, z_{m}^{\prime} \in\left|f_{m}\left(C_{m}^{2}\right)\right|$, where $z_{m}, z_{m}^{\prime} \rightarrow z_{0} \in V$ as $m \rightarrow \infty$. In this case, $\left|f_{m}\left(C_{m}^{1}\right)\right| \cap V \neq \varnothing \neq\left|f_{m}\left(C_{m}^{2}\right)\right| \cap V$ for sufficiently large $m \in \mathbf{N}$. Also, $h(V) \leqslant h(U) \leqslant 2 r_{0}<\delta_{1} / 2$. Further, by (4.23) we obtain that $h\left(\left|f_{m}\left(C_{m}^{1}\right)\right|\right)>\delta_{1} / 2$. Therefore, $\left|f_{m}\left(C_{m}^{1}\right)\right| \cap\left(D^{\prime} \backslash V\right) \neq \varnothing$ and, therefore, $\left|f_{m}\left(C_{m}^{1}\right)\right| \cap \partial V \neq \varnothing$ (see $[\mathrm{Ku}$, Theorem 1.I.5.46]). Similarly, $h(V) \leqslant h(U) \leqslant 2 r_{0}<\delta_{1} / 2$. By $(4.23) h\left(\left|f_{m}\left(C_{m}^{2}\right)\right|\right)>$ $\delta_{1} / 2$, therefore $\left|f_{m}\left(C_{m}^{2}\right)\right| \cap\left(D^{\prime} \backslash V\right) \neq \varnothing$. By [Ku, Theorem 1.I.5.46] we obtain that $\left|f_{m}\left(C_{m}^{2}\right)\right| \cap \partial V \neq \varnothing$. Thus, the relation (4.26) is established.

Combining the relations (4.24), (4.25) and (4.26), we obtain that

$$
M\left(f_{m}\left(\Gamma_{m}\right)\right)=M\left(\Gamma\left(\left|f_{m}\left(C_{m}^{1}\right)\right|,\left|f_{m}\left(C_{m}^{2}\right)\right|, D^{\prime}\right)\right)>P .
$$

The last relation contradicts the inequality (4.22). Theorem is completely proved.

Remark 4.27. One of the versions of Theorem 1.6 was established by us earlier and related to less general classes of mappings, see [SevSkv, Theorem 3]. In addition, the mapped domain $D^{\prime}$ here was $Q E D$-domain by Gehring-Martio, which can also be considered as a particular case of a domain with a weakly flat boundary. We also mention paper [SSI], where the boundary behavior of families of mappings with two normalization conditions is investigated. Although the publications mentioned above correspond to the situation of general metric spaces, the results of this article do not follow from them in full. In particular, an equicontinuity of families of mappings inside a domain cannot be obtained as in Theorem 1.5, in an arbitrary metric space.

\section{On isolated singularities of inverse mappings}

We consider the removability of an isolated singularity for mappings whose inverses satisfy (1.1). Isolated singularities have been studied in [MRSY ${ }_{2}$, Corollary 5.23], [RS, Theorem 6.1] and [Sm, Theorem 5], but, besides (1.1), we do not assume any extra conditions on the domains of definitions and their images. The main result is as follows. 
Theorem 5.1. Let $D$ and $D^{\prime}$ be domains in $\overline{\mathbf{R}^{n}}, n \geqslant 2$, and let $g$ be a homeomorphism of a domain $D^{\prime}$ onto a domain $D$, the inverse $f=g^{-1}$ of which satisfies the condition (1.1) at every point $x_{0} \in \partial D$. If $Q \in L^{1}(D)$ and $y_{0}$ is an isolated point of the boundary of the domain $D^{\prime}$, then the mapping $g$ has a continuous extension $\bar{g}: D^{\prime} \cup\left\{y_{0}\right\} \rightarrow \overline{\mathbf{R}^{n}}$ to $y_{0}$.

Theorem 5.1 does not imply the removability of an isolated singularity for $f$, see [MRSY ${ }_{3}$, Proposition 6.3], although for quasiconformal mappings, as in the particular case of such classes, we can still assert the validity of Theorem 5.1 for both $f$ and $g$, see [ $\mathrm{Va}_{1}$, Theorem 17.3]. As before, the boundary, closure, and continuous extension of maps are understood in the sense of the extended space $\overline{\mathbf{R}^{n}}$. For a given mapping $f: D \rightarrow \overline{\mathbf{R}^{n}}$ and a set $E$ in $\overline{\mathbf{R}^{n}}$, we put

$$
C(f, E)=\left\{y \in \overline{\mathbf{R}^{n}}: \exists x \in E, x_{k} \in D: x_{k} \rightarrow x, f\left(x_{k}\right) \rightarrow y, k \rightarrow \infty\right\} .
$$

The next most important statement is published in [IR, Lemma 5.3], see also [MRSY ${ }_{3}$, Lemma 6.5].

Proposition 5.2. Let $D$ and $D^{\prime}$ be domains in $\overline{\mathbf{R}^{n}}, n \geqslant 2$, and $f: D \rightarrow \overline{\mathbf{R}^{n}}$ a homeomorphism. Then there exists a one-to-one correspondence between the components $K$ and $K^{\prime}$ of the boundaries $\partial D$ and $\partial D^{\prime}$ such that $C(f, K)=K^{\prime}$ and $C\left(f^{-1}, K^{\prime}\right)=K$.

Proof of Theorem 5.1. Without loss of generality, we may assume that $y_{0} \neq$ $\infty$. Here and everywhere, as usual, $h(x, y)$ denotes the chordal (spherical) distance between points $x, y \in \overline{\mathbf{R}^{n}}$, see (1.3). Suppose the contrary, namely, suppose that $g$ has no limit at $y_{0}$. Since the space $\overline{\mathbf{R}^{n}}$ is compact, $C\left(g, y_{0}\right) \neq \varnothing$. Then there are $x_{1}, x_{2} \in \overline{\mathbf{R}^{n}}, x_{1} \neq x_{2}$, and at least two sequences $y_{m}, y_{m}^{\prime} \rightarrow y_{0}$ as $m \rightarrow \infty$ such that $z_{m}:=g\left(y_{m}\right) \rightarrow x_{1}, z_{m}^{\prime}=g\left(y_{m}^{\prime}\right) \rightarrow x_{2}$ as $m \rightarrow \infty$. Without loss of generality, we may assume that $x_{1} \neq \infty$.

Since $y_{0} \in \partial D^{\prime}$ is an isolated point of $\partial D^{\prime}, C\left(g, y_{0}\right)$ is a continuum and, moreover, is a component of the boundary $\partial D$ (see Proposition 5.2). Let us show that there exists $\varepsilon_{1}>0$ such that

$$
B\left(x_{1}, \varepsilon_{1}\right) \cap K=\varnothing
$$

for every component $K$ of the boundary $\partial D$ such that $C\left(g, y_{0}\right) \neq K$.

We establish the relation (5.3) also by contradiction. Suppose the contrary. Then there is a sequence $d_{m}$ of components of $\partial D, d_{m} \neq C\left(g, y_{0}\right), m=1,2, \ldots$, such that $B\left(x_{1}, 1 / m\right) \cap d_{m} \neq \varnothing$. By Proposition 5.2, the component $d_{m}$ of the set $\partial D$ is in one-to-one correspondence with some component $d_{m}^{\prime} \subset \partial D^{\prime}$ such that $C\left(f, d_{m}\right)=d_{m}^{\prime}$. Therefore, we may choose $\zeta_{m} \in B\left(x_{1}, 1 / m\right) \cap D$ so that

$$
h\left(f\left(\zeta_{m}\right), d_{m}^{\prime}\right)=\inf _{p \in d_{m}^{\prime}} h\left(f\left(\zeta_{m}\right), p\right)<1 / m
$$

Since $d_{m}^{\prime}$ is a compact set in $\overline{\mathbf{R}^{n}}$, there is $\xi_{m} \in d_{m}^{\prime}$ such that $h\left(f\left(\zeta_{m}\right), d_{m}^{\prime}\right)=$ $h\left(f\left(\zeta_{m}\right), \xi_{m}\right)$. Since $\overline{\mathbf{R}^{n}}$ is compact, there is a subsequence $f\left(\zeta_{m_{k}}\right)$, converging in $\overline{\mathbf{R}^{n}}$ as $k \rightarrow \infty$. Since $\zeta_{m_{k}} \in B\left(x_{1}, 1 / m_{k}\right)$, then by Proposition 5.2 the sequence $f\left(\zeta_{m_{k}}\right)$ can converge only to $y_{0}$ as $k \rightarrow \infty$. Then by the triangle inequality

$$
h\left(y_{0}, d_{m_{k}}^{\prime}\right) \leqslant h\left(y_{0}, \xi_{m_{k}}\right) \leqslant h\left(y_{0}, f\left(\zeta_{m_{k}}\right)\right)+h\left(f\left(\zeta_{m_{k}}\right), \xi_{m_{k}}\right) \rightarrow 0
$$

as $k \rightarrow \infty$, which contradicts the assumption that the point $y_{0}$ is an isolated point of $\partial D^{\prime}$. We may consider that $f(x) \neq \infty$ for $x \in B\left(x_{1}, \varepsilon_{1}\right) \cap D$. Let $B_{*}\left(x_{2}, \varepsilon_{2}\right)=$ 
$B\left(x_{2}, \varepsilon_{2}\right)$ for $x_{2} \neq \infty$ and $B_{*}\left(x_{2}, \varepsilon_{2}\right)=\left\{x \in \overline{\mathbf{R}^{n}}: h(x, \infty)<\varepsilon_{2}\right\}$ for $x_{2}=\infty$. As in the proof for (5.3) there is $\varepsilon_{2}>0$ such that

$$
B_{*}\left(x_{2}, \varepsilon_{2}\right) \cap K=\varnothing
$$

for every component $K$ of the boundary $\partial D$, not equal to $C\left(g, y_{0}\right) \neq K$. Without loss of generality, we may assume that $\overline{B\left(x_{1}, \varepsilon_{1}\right)} \cap \overline{B_{*}\left(x_{2}, \varepsilon_{2}\right)}=\varnothing, z_{m} \in B\left(x_{1}, \varepsilon_{1}\right)$ and $z_{m}^{\prime} \in B_{*}\left(x_{2}, \varepsilon_{2}\right)$ for all $m=1,2, \ldots$ (see Figure 4$)$.

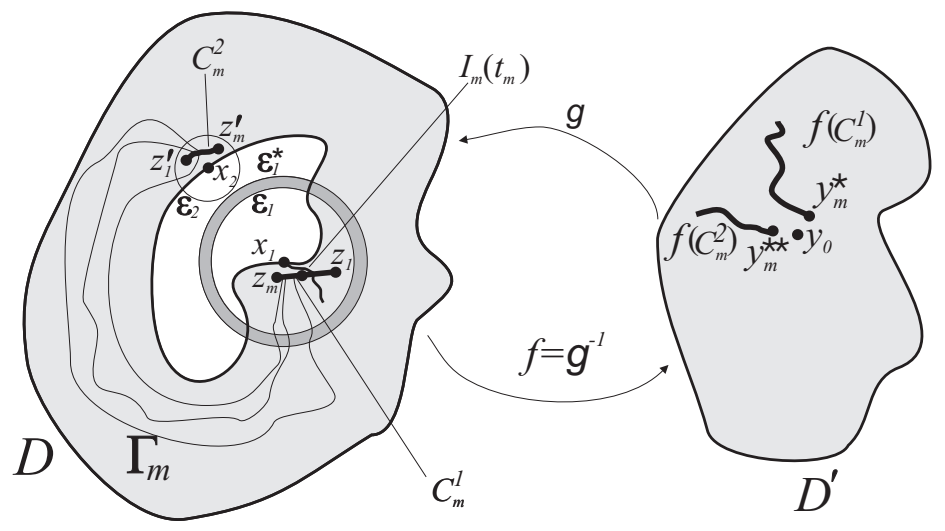

Figure 4. To the proof of Theorem 5.1.

Note that the set $B\left(x_{1}, \varepsilon_{1}\right)$ is convex, and $B_{*}\left(x_{2}, \varepsilon_{2}\right)$ is path connected. In this case, the points $z_{1}$ and $z_{m}$ can be joined by the segment $I_{m}(t)=z_{1}+t\left(z_{m}-z_{1}\right)$, $t \in(0,1)$, lying inside the ball $B\left(x_{1}, \varepsilon_{1}\right)$. Similarly, the points $z_{1}^{\prime}$ and $z_{m}^{\prime}$ can be connected by the path $J_{m}=J_{m}(t), t \in[0,1]$, lying in the "ball" $B_{*}\left(x_{2}, \varepsilon_{2}\right)$.

Note that the set $\left|I_{m}\right|$ does not have to lie in the domain $D$ entirely. However, in this case, there is $t_{m} \in[0,1]$ such that $h\left(I_{m}\left(t_{m}\right), \partial D\right)<1 / m$ and $\left|I_{m}\right|_{\left[0, t_{m}\right]} \mid \subset$ $D$. Similarly, $J_{m}$ may not be in $D$ entirely, but there is $p_{m} \in[0,1]$ such that $h\left(J_{m}\left(p_{m}\right), \partial D\right)<1 / m$ and $\left|J_{m}\right|_{\left[0, p_{m}\right]} \mid \subset D$. If $I_{m} \subset D$ or $J_{m} \subset D$, then we put $t_{m}:=1$ and $p_{m}:=1$, respectively. Put $C_{m}^{1}:=\left.I_{m}\right|_{\left[0, t_{m}\right]}$ and $C_{m}^{2}:=\left.J_{m}\right|_{\left[0, p_{m}\right]}$. Consider the sequences $y_{m}^{*}:=f\left(I_{m}\left(t_{m}\right)\right)$ and $y_{m}^{* *}:=f\left(J_{m}\left(p_{m}\right)\right)$. Since the space $\overline{\mathbf{R}^{n}}$ is compact, we may assume that all considered sequences $I_{m}\left(t_{m}\right), J_{m}\left(p_{m}\right), y_{m}^{*}$ and $y_{m}^{* *}$ converge as $m \rightarrow \infty$.

We show that $y_{m}^{*} \rightarrow y_{0}$ and $y_{m}^{* *} \rightarrow y_{0}$ as $m \rightarrow \infty$. Indeed, let $y_{m}^{*} \rightarrow w_{0}$ as $m \rightarrow \infty$. Since the sequence $I_{m}\left(t_{m}\right)$ converges by assumption and, moreover, $h\left(I_{m}\left(t_{m}\right), \partial D\right)<$ $1 / m$, then $I_{m}\left(t_{m}\right)$ converges to some point $\omega_{0} \in \partial D$. Since $I_{m}\left(t_{m}\right) \in B\left(x_{1}, \varepsilon_{1}\right)$, and, moreover, by $(5.3)$ the ball $B\left(x_{1}, \varepsilon_{1}\right)$ does not contain other components of $\partial D$ besides $C\left(g, y_{0}\right)$, we have that $\omega_{0} \in C\left(g, y_{0}\right)$. Since $y_{m}^{*}=f\left(I_{m}\left(t_{m}\right)\right)$ and $y_{m}^{*} \rightarrow y_{0}$ as $m \rightarrow \infty$, we obtain that $w_{0} \in C\left(f, C\left(g, y_{0}\right)\right)$. Taking into account that $g=f^{-1}$, by Proposition 5.2 we obtain that $w_{0}=y_{0}$, as required. Reasoning similarly, we may show that $y_{m}^{* *} \rightarrow y_{0}$ as $m \rightarrow \infty$.

Since $\overline{B\left(x_{1}, \varepsilon_{1}\right)} \cap \overline{B_{*}\left(x_{2}, \varepsilon_{2}\right)}=\varnothing$, then for some $\varepsilon_{1}^{*}>\varepsilon_{1}$ we also still have $\overline{B\left(x_{1}, \varepsilon_{1}^{*}\right)} \cap \overline{B_{*}\left(x_{2}, \varepsilon_{2}\right)}=\varnothing$. We may consider that $f(x) \neq \infty$ for $x \in B\left(x_{1}, \varepsilon_{1}^{*}\right) \cap D$. Let $\Gamma_{m}=\Gamma\left(\left|C_{m}^{1}\right|,\left|C_{m}^{2}\right|, D\right)$. Note that

$$
\Gamma_{m}>\Gamma\left(S\left(x_{1}, \varepsilon_{1}^{*}\right), S\left(x_{1}, \varepsilon_{1}\right), A\left(x_{1}, \varepsilon_{1}, \varepsilon_{1}^{*}\right) \cap D\right) .
$$

Indeed, let $\gamma \in \Gamma_{m}, \gamma:[a, b] \rightarrow \mathbf{R}^{n}$. Since $\gamma(a) \in\left|C_{m}^{1}\right| \subset B\left(x_{0}, \varepsilon_{1}\right)$ and $\gamma(b) \in$ $\left|C_{m}^{2}\right| \subset \mathbf{R}^{n} \backslash B\left(x_{0}, \varepsilon_{1}\right)$, due to [Ku, Theorem 1.I.5.46] there is $t_{1} \in(a, b)$ such that $\gamma\left(t_{1}\right) \in S\left(x_{1}, \varepsilon_{1}\right)$. Without loss of generality, we may assume that $\left|\gamma(t)-x_{1}\right|>\varepsilon_{1}$ for $t>t_{1}$. Next, since $\gamma\left(t_{1}\right) \in B\left(x_{1}, \varepsilon_{1}^{*}\right)$ and $\gamma(b) \in\left|C_{m}^{2}\right| \subset \mathbf{R}^{n} \backslash B\left(x_{0}, \varepsilon_{1}^{*}\right)$, by $[\mathrm{Ku}$, 
Theorem 1.I.5.46] there is $t_{2} \in\left(t_{1}, b\right)$ such that $\gamma\left(t_{2}\right) \in S\left(x_{1}, \varepsilon_{1}^{*}\right)$. Without loss of generality, we may assume that $\left|\gamma(t)-x_{1}\right|<\varepsilon_{1}^{*}$ for $t_{1}<t<t_{2}$. Thus, $\left.\gamma\right|_{\left[t_{1}, t_{2}\right]}$ is a subpath of $\gamma$, belonging to the family $\Gamma\left(S\left(x_{1}, \varepsilon_{1}^{*}\right), S\left(x_{1}, \varepsilon_{1}\right), A\left(x_{1}, \varepsilon_{1}, \varepsilon_{1}^{*}\right)\right)$. Thus, the relation (5.4) is established. Consider the function

$$
\eta(t)= \begin{cases}1 /\left(\varepsilon_{1}^{*}-\varepsilon_{1}\right), & t \in\left[\varepsilon_{1}, \varepsilon_{1}^{*}\right], \\ 0, & t \in \mathbf{R} \backslash\left[\varepsilon_{1}, \varepsilon_{1}^{*}\right] .\end{cases}
$$

Note that the function $\eta$ satisfies the relation (1.2) for $r_{1}=\varepsilon_{1}$ and $r_{2}=\varepsilon_{1}^{*}$. By (1.1) at $x_{0}:=x_{1}$, taking into account the conditions $Q \in L^{1}(D)$ and (5.4), we obtain that

$$
\begin{aligned}
M\left(f\left(\Gamma_{m}\right)\right) & \leqslant M\left(f\left(\Gamma\left(S\left(x_{1}, \varepsilon_{1}^{*}\right), S\left(x_{1}, \varepsilon_{1}\right), A\left(x_{1}, \varepsilon_{1}, \varepsilon_{1}^{*}\right) \cap D\right)\right)\right) \\
& \leqslant\|Q\| /\left(\varepsilon_{1}^{*}-\varepsilon_{1}\right)^{n}<\infty
\end{aligned}
$$

where $\|Q\|$ denotes $L^{1}$-norm of the function $Q$ in $D$. We show that the relation (5.5) contradicts the condition of a weak flatness at the point $y_{0}$ (see Lemma 2.2). Indeed,

$$
\operatorname{diam}\left|f\left(C_{m}^{1}\right)\right| \geqslant\left|f\left(z_{1}\right)-f\left(I_{m}\left(t_{m}\right)\right)\right|=\left|y_{1}-y_{m}^{*}\right| \geqslant(1 / 2) \cdot\left|y_{1}-y_{0}\right|>0
$$

and

$$
\operatorname{diam}\left|f\left(C_{m}^{2}\right)\right| \geqslant\left|f\left(z_{1}^{\prime}\right)-f\left(J_{m}\left(p_{m}\right)\right)\right|=\left|y_{1}^{\prime}-y_{m}^{* *}\right| \geqslant(1 / 2) \cdot\left|y_{1}^{\prime}-y_{0}\right|>0
$$

for large $m \in \mathbf{N}$, and, in addition,

$$
\operatorname{dist}\left(\left|f\left(C_{m}^{1}\right)\right|,\left|f\left(C_{m}^{2}\right)\right|\right) \leqslant\left|y_{m}^{*}-y_{m}^{* *}\right| \rightarrow 0
$$

as $m \rightarrow \infty$. By Lemma 2.2

$$
M\left(f\left(\Gamma_{m}\right)\right)=M\left(\Gamma\left(\left|f\left(C_{m}^{1}\right)\right|,\left|f\left(C_{m}^{2}\right)\right|, D^{\prime}\right)\right) \rightarrow \infty
$$

as $m \rightarrow \infty$, which contradicts the relation (5.5).

Remark 5.6. It is easy to see that the assertion of Theorem 5.1 is true under much weaker condition on the mapping $f$, namely, it is enough to require the condition (1.1) just in one finite point of the cluster set $C\left(g, y_{0}\right)$.

Moreover, it is not so important whether to require the condition (1.1) in $\partial D$ or in $D$. In fact, suppose that under conditions of Theorem 5.1 we require the relation (1.1) not on $\partial D$, but at each inner point $x_{0} \in D$. Repeating the proof of this theorem at the same notations, we obtain the relation (5.4). Now let $a_{k} \in D$, $k=1,2, \ldots$, be some (arbitrary) sequence of points converging to $x_{1}$ as $k \rightarrow \infty$ such that $\left|a_{k}-x_{1}\right|<1 / k$. Fix $x \in B\left(x_{1}, \varepsilon_{1}\right)$. Then, by the triangle inequality $\left|x-a_{k}\right| \leqslant\left|x-x_{1}\right|+\left|x_{1}-a_{k}\right|<\varepsilon_{1}+1 / k$ and, therefore, $B\left(x_{1}, \varepsilon_{1}\right) \subset B\left(a_{k}, \varepsilon_{1}+1 / k\right)$. Further, for $x \in B\left(a_{k}, \varepsilon_{1}+2 / k\right)$ by the triangle inequality we have that

$$
\left|x-x_{1}\right| \leqslant\left|x-a_{k}\right|+\left|a_{k}-x_{1}\right|<\varepsilon_{1}+3 / k .
$$

Let $k_{0} \in \mathbf{N}$ be so large that $\varepsilon_{1}+3 / k<\varepsilon_{1}^{*}$ for $k>k_{0}$. Then $B\left(a_{k}, \varepsilon_{1}+2 / k\right) \subset B\left(x_{1}, \varepsilon_{1}^{*}\right)$ for $k>k_{0}$. Putting $\widetilde{\varepsilon_{1}}:=\varepsilon_{1}+1 /\left(k_{0}+1\right)$ and $\widetilde{\varepsilon_{2}}:=\varepsilon_{1}+2 /\left(k_{0}+1\right)$, we obtain that

$$
B\left(x_{1}, \varepsilon_{1}\right) \subset B\left(a_{k_{0}+1}, \widetilde{\varepsilon_{1}}\right) \subset B\left(a_{k_{0}+1}, \widetilde{\varepsilon_{2}}\right) \subset B\left(x_{1}, \varepsilon_{1}^{*}\right) .
$$

Arguing by analogy with the proof of the formula (5.4), from the relation (5.7) we obtain that

$$
\begin{aligned}
& \Gamma\left(S\left(x_{1}, \varepsilon_{1}^{*}\right), S\left(x_{1}, \varepsilon_{1}\right), A\left(x_{1}, \varepsilon_{1}, \varepsilon_{1}^{*}\right) \cap D\right) \\
& >\Gamma\left(S\left(a_{k_{0}+1}, \widetilde{\varepsilon_{1}}\right), S\left(a_{k_{0}+1}, \widetilde{\varepsilon_{2}}\right), A\left(a_{k_{0}+1}, \widetilde{\varepsilon_{1}}, \widetilde{\varepsilon_{2}}\right) \cap D\right),
\end{aligned}
$$

see Figure 5. 


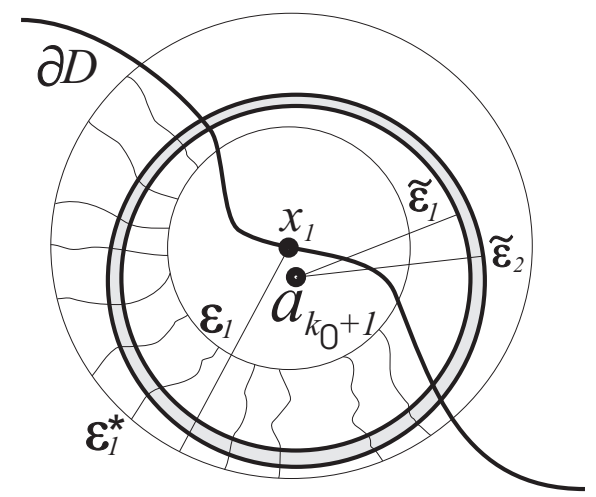

Figure 5. To Remark 5.6.

Consider the function

$$
\eta(t)= \begin{cases}1 /\left(\widetilde{\varepsilon_{2}}-\widetilde{\varepsilon_{1}}\right), & t \in\left[\widetilde{\varepsilon_{1}}, \widetilde{\varepsilon_{2}}\right], \\ 0, & t \in \mathbf{R} \backslash\left[\widetilde{\varepsilon_{1}}, \widetilde{\varepsilon_{2}}\right] .\end{cases}
$$

Note that the function $\eta$ satisfies the relation (1.2) with $r_{1}=\widetilde{\varepsilon_{1}}$ and $r_{2}=\widetilde{\varepsilon_{2}}$. Then by (1.1) at $x_{0}:=a_{k_{0}+1}$, taking into account the condition $Q \in L^{1}(D)$ and the relations (5.4) and (5.8), we obtain that

$$
\begin{aligned}
M\left(f\left(\Gamma_{m}\right)\right) & \leqslant M\left(f\left(\Gamma\left(S\left(a_{k_{0}+1}, \widetilde{\varepsilon_{1}}\right), S\left(a_{k_{0}+1}, \widetilde{\varepsilon_{2}}\right), A\left(a_{k_{0}+1}, \widetilde{\varepsilon_{1}}, \widetilde{\varepsilon_{2}}\right) \cap D\right)\right)\right) \\
& \leqslant\|Q\| /\left(\widetilde{\varepsilon_{2}}-\widetilde{\varepsilon_{1}}\right)^{n}<\infty,
\end{aligned}
$$

where $\|Q\|$ denotes $L^{1}$-norm of $Q$ in $D$. So, instead of (5.5) we have the relation (5.9). The rest of the proof of Theorem 5.1, based on a contradiction of (5.9) with the weak flatness condition at the point $y_{0}$, does not change.

\section{Some examples}

Example 6.1. We have already mentioned that maps inverse to a given class may turn out to be better (or worse) than the original class of maps. This situation is impossible for quasiconformal mappings, but is quite real for relatively simple mappings with unbounded characteristic. Here is one such example.

Fix a number $p \geqslant 1$ satisfying the condition $n / p(n-1)<1$. Put $\alpha \in(0, n / p(n-$ $1)$ ). We define the sequence of mappings $f_{m}$ of the unit ball onto the ball $B(0,2)$ as follows:

$$
f_{m}(x)= \begin{cases}\frac{1+|x|^{\alpha}}{|x|} \cdot x, & 1 / m \leqslant|x| \leqslant 1 \\ \frac{1+(1 / m)^{\alpha}}{(1 / m)} \cdot x, & 0<|x|<1 / m\end{cases}
$$

Note that $f_{m}$ satisfies the condition (1.1) for $Q=\left(\frac{1+|x|^{\alpha}}{\alpha|x|^{\alpha}}\right)^{n-1}$ at every $x_{0} \in \overline{\mathbf{B}^{n}}$, moreover, $Q \in L^{p}\left(\mathbf{B}^{n}\right)$ see, for example, [Sev, proof of Theorem 7.1].

By [Vu, Lemma 4.3], the ball $B(0,2)$ has a weakly flat boundary. Note that the mappings $f_{m}$ fix an infinite number of points of the unit ball for every $m \geqslant 2$. By Theorem 1.6, the family $\mathfrak{G}=\left\{g_{m}\right\}_{m=1}^{\infty}, g_{m}:=f_{m}^{-1}$, is equicontinuous in $\overline{B(0,2)}$.

Note that the "inverse" family of mappings $\mathfrak{F}=\left\{f_{m}\right\}_{m=1}^{\infty}$ is not equicontinuous in $\mathbf{B}^{n}$. Indeed, $\left|f_{m}\left(x_{m}\right)-f_{m}(0)\right|=1+1 / m^{\alpha} \rightarrow 1$ as $m \rightarrow \infty$, where $\left|x_{m}\right|=1 / m$. In particular, it follows that the family $\mathfrak{G}$ contains an infinite number of mappings $g_{m_{k}}:=f_{m_{k}}^{-1}, f_{m_{k}} \in \mathfrak{F}$, that cannot satisfy the relation (1.1) for any functions $Q \in L^{1}$. Indeed, otherwise, by Theorem 1.5, $\mathfrak{F}$ must be equicontinuous in $\mathbf{B}^{n}$. 
Example 6.2. As for Theorem 5.1, we can also construct an example that is very similar to the one already given above. More precisely, let us point out the case when the inverse mapping extends by continuity to an isolated point of the boundary of the domain, and the corresponding direct mapping does not already have this property.

Let $p \geqslant 1, \alpha \in(0, n / p(n-1))$ and $e_{1}=(0,0, \ldots, 0,1 / 2)$. Consider the mapping $f$ in the domain $D:=\mathbf{B}^{n} \backslash\left\{e_{1} \cup 0\right\}$ as follows:

$$
f(x)=\frac{1+|x|^{\alpha}}{|x|} \cdot x, \quad x \in \mathbf{B}^{n} \backslash\left\{e_{1} \cup 0\right\} .
$$

It is easy to see that the mapping $f$ is a ring $Q$-mapping of $\mathbf{B}^{n} \backslash\left\{e_{1} \cup 0\right\}$ onto $A:=$ $\{1<|y|<2\} \backslash\left\{e_{2}\right\}$, where $Q(x):=\left(\frac{1+r^{\alpha}}{\alpha^{\alpha}}\right)^{n-1}, r=|x|$ (see, e.g., [MRSY 3 , Proposition 6.3]). Moreover, $Q \in L^{p}\left(\mathbf{B}^{n}\right)$. Note that $f\left(e_{1}\right)=\left(0,0, \ldots, 0,1+\left(\frac{1}{2}\right)^{\alpha}\right):=e_{2}$. The inverse mapping $g:=f^{-1}(y)=\frac{y}{|y|}(|y|-1)^{1 / \alpha}$ has a continuous extension to the point $e_{2}, \bar{g}:=\frac{y}{|y|}(|y|-1)^{1 / \alpha}, \bar{g}:\{1<|y|<2\} \rightarrow \mathbf{B}^{n} \backslash\{0\}$ (the existence of this extension also follows from Theorem 5.1). On the other hand, the mapping $\bar{f}:=\overline{g^{-1}}$, $\bar{f}: \mathbf{B}^{n} \backslash\{0\} \rightarrow\{1<|y|<2\}$, does not have a continuous extension to the point 0 , which is an isolated point of the boundary of the domain $\mathbf{B}^{n} \backslash\{0\}$. The latter circumstance is connected with the non-existence of an integrable function $Q^{*}(y)$, corresponding to the map $\bar{g}$ in $\{1<|y|<2\}$ in the context of the inequality (1.1).

\section{References}

[Cr] CRistea, M.: Open discrete mappings having local $A C L^{n}$ inverses. - Complex Var. Elliptic Equ. 55:1-3, 2010, 61-90.

[GU] Gol'Dshtein, V., and A. Ukhlov: Traces of functions of $L_{2}^{1}$ Dirichlet spaces on the Carathéodory boundary. - Studia Math. 235:3, 2016, 209-224.

[HK] Herron, D. A., and P. Koskela: Quasiextremal distance domains and conformal mappings onto circle domains. - Complex Variables Theory Appl. 15, 1990, 167-179.

[HW] Hurewicz, W., and H. Wallman: Dimension theory. - Princeton Univ. Press, Princeton, 1948.

[IR] Ignat'ev, A., and V. RyazAnov: Finite mean oscillation in mapping theory. - Ukr. Mat. Visn. 2:3, 2005, 395-417 (in Russian); English transl. in Ukr. Math. Bull. 2:3, 2005, 403-424.

[Keld] Keldysh, L.V.: Topological imbeddings in Euclidean space. - Trudy MIAN SSSR, 81, 1966, 3-184 (in Russian); English transl. in Proc. Steklov Inst. Math. 81, 1966, 1-203.

[KO] Koskela, P., and J. Onninen: Mappings of finite distortion: Capacity and modulus inequalities. - J. Reine Angew. Math. 599, 2006, 1-26.

[Ku] Kuratowski, K.: Topology. Volume 2. - Academic Press, New York-London, 1968.

[MRV] Martio, O., S. Rickman, and J. VÄIsÄlä: Distortion and singularities of quasiregular mappings. - Ann. Acad. Sci. Fenn. Ser. A I Math. 465, 1970, 1-13.

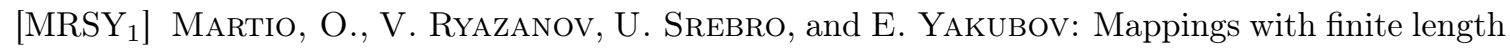
distortion. - J. Anal. Math. 93, 2004, 215-236.

[MRSY 2 ] Martio, O., V. Ryazanov, U. Srebro, and E. Yakubov: On $Q$-homeomorphisms. Ann. Acad. Sci. Fenn. Math. 30:1, 2005, 49-69.

[MRSY ${ }_{3}$ ] Martio, O., V. Ryazanov, U. Srebro, and E. Yakubov: Moduli in modern mapping theory. - Springer Monogr. Math., Springer, New York, 2009.

[NP 1 NÄKKI, R., and B. PALKA: Uniform equicontinuity of quasiconformal mappings. - Proc. Amer. Math. Soc. 37:2, 1973, 427-433. 
$\left[\mathrm{NP}_{2}\right]$ NÄKKI, R., and B. PALKA: Boundary regularity and the uniform convergence of quasiconlormal mappings. - Comment. Math. Helv. 54, 1979, 458-476.

[Pol] Poletskil, E. A.: The modulus method for non-homeomorphic quasiconformal mappings. - Mat. Sb. 83:2, 1970, 261-272 (in Russian).

[RS] Ryazanov, V., and R. Salimov: Weakly planar spaces and boundaries in the theory of mappings. - Ukr. Mat. Visn. 4:2, 2007, 199-234 (in Russian); English transl. in Ukr. Math. Bull. 4:2, 2007, 199-234.

[RSY] Ryazanov, V., U. SRebro, and E. Yakubov: On ring solutions of Beltrami equations. - J. Anal. Math. 96, 2005, 117-150.

[Sev] SEvost'Yanov, E.: On the equicontinuity of homeomorphisms with an unbounded characteristic. - Mat. Trudy 15:1, 2012, 178-204 (in Russian); English transl. in Siberian Adv. Math. 23:2, 2013, 106-122.

[SevSkv] Sevost'yanov, E. A., and S. A. Skvortsov: On the convergence of mappings in metric spaces with direct and inverse modulus conditions. - Ukr. Mat. Zh., 70:7, 2018, 952-967 (in Russian); English transl. in Ukr. Math. Journ., 70:7, 2018, 1097-1114.

[SSI] Sevost'yanov, E. A., S. A. Skvortsov, and N. S. Ilkevych: On boundary behavior of mappings with two normalized conditions. - Mat. Stud. 49:2, 2018, 150-157.

[Sm] Smolovaya, E. S.: Boundary behavior of ring $Q$-homeomorphisms in metric spaces. Ukr. Mat. Zh. 62:5, 2010, 682-689 (in Russian); English transl. in Ukrainian Math. J. 62:5, 2010, 785-793.

[Va 1 VÄISÄLÄ J.: Lectures on $n$-dimensional quasiconformal mappings. - Lecture Notes in Math. 229, Springer-Verlag, Berlin etc., 1971.

[Va 2 VÄISÄLÄ, J.: Modulus and capacity inequalities for quasiregular mappings. - Ann. Acad. Sci. Fenn. Ser. A I Math. 509, 1972, 1-14.

[Vu] Vuorinen, M.: On the existence of angular limits of $n$-dimensional quasiconformal mappings. - Ark. Mat. 18, 1980, 157-180.

Received 3 January 2019 • Revised received 13 March 2019 • Accepted 26 March 2019 